\title{
An aluminium tool for multiple stellar generations in the globular clusters 47 Tucanae and $M 4^{\star, \star \star}$
}

\author{
E. Carretta ${ }^{1}$, R. G. Gratton ${ }^{2}$, A. Bragaglia ${ }^{1}$, V. D’Orazi ${ }^{3,4}$, and S. Lucatello ${ }^{2}$ \\ 1 INAF - Osservatorio Astronomico di Bologna, via Ranzani 1, 40127 Bologna, Italy \\ e-mail: eugenio.carretta@oabo.inaf.it \\ 2 INAF - Osservatorio Astronomico di Padova, Vicolo dell'Osservatorio 5, 35122 Padova, Italy \\ 3 Department of Physics and Astronomy, Macquarie University, Balaclava rd, North Ryde, NSW 2109, Australia \\ 4 Monash Centre for Astrophysics, School of Mathematical Sciences, Building 28, Monash University, VIC 3800, Australia
}

Received 1 October 2012 / Accepted 2 December 2012

\begin{abstract}
We present aluminium abundances for a sample of about 100 red giant stars in each of the Galactic globular clusters 47 Tuc (NGC 104) and M 4 (NGC 6121). We have derived homogeneous abundances from intermediate-resolution FLAMES/GIRAFFE spectra. Aluminium abundances are from the strong doublet Al I 8772-8773 $\AA$, as in previous works done for giants in NGC 6752 and NGC 1851, and nitrogen abundances are extracted from a large number of features of the CN molecules by assuming a suitable carbon abundance. We added previous homogeneous abundances of $\mathrm{O}$ and $\mathrm{Na}$ and newly derived abundances of $\mathrm{Mg}$ and $\mathrm{Si}$ for our samples of 83 stars in M 4 and 116 stars in 47 Tuc to obtain the full set of elements from proton-capture reactions produced by different stellar generations in these clusters. By simultaneously studying the $\mathrm{Ne}-\mathrm{Na}$ and $\mathrm{Mg}$ - $\mathrm{Al}$ cycles of $\mathrm{H}$-burning at high temperature, our main aims are to understand the nature of the polluters at work in the first generation and to ascertain whether the second generation of cluster stars was formed in one or, rather, several episodes of star formation. Our data confirm that in M 4 only two stellar populations are visible. On the other hand, for 47 Tuc a cluster analysis performed on our full dataset suggests that at least three distinct groups of stars are present on the giant branch. The abundances of $\mathrm{O}, \mathrm{Na}, \mathrm{Mg}$, and $\mathrm{Al}$ in the intermediate group can be produced within a pollution scenario; results for $\mathrm{N}$ are ambiguous, depending on the $\mathrm{C}$ abundance we adopt for the three groups.
\end{abstract}

Key words. stars: abundances - stars: atmospheres - stars: Population II - globular clusters: individual: 47 Tucanae globular clusters: general - globular clusters: individual: M4

\section{Introduction}

The new paradigm for globular cluster (GCs) formation is currently well assessed in its general aspects. Several decades of spectroscopic observations as well as years of high-precision imaging, mainly with the Hubble Space Telescope (HST), have shown that a typical pattern is present within each galactic GC investigated so far (see the recent reviews by Gratton et al. 2004; Martell 2011; Gratton et al. 2012, and references therein). A first generation of stars is found side by side with another, slightly younger stellar generation, whose components are unambiguously formed by ejecta processed in the nuclear H-burning at high temperature within massive stars of the first generation in each cluster (see, e.g., Gratton et al. 2001, for the first clear-cut observational constraint; followed by Ramirez \& Cohen 2002; Carretta et al. 2004; see, e.g., Ventura et al. 2001 and Decressin et al. 2007 for different views on the nature of these early polluters of the intra-cluster medium).

The concept of GCs being good examples of simple stellar populations (SSP) has been eventually replaced by their being made of multiple stellar populations (MSP), whose compositions are marked by a typical pattern: light elements show anti-correlations and correlations among species produced (such

* Based on observations collected at ESO telescopes under program 085.D-0205 and on public data from the ESO/ST-ECF Science Archive Facility.

$\star \star$ Tables 2 and 3 are available in electronic form at http://www. aanda.org as $\mathrm{Na}, \mathrm{Al}, \mathrm{Si}$ ) and destroyed (such as $\mathrm{O}, \mathrm{Mg}, \mathrm{F}$ ) in the network of proton-capture reactions responsible for the nuclear processing of the polluting matter (e.g., Denisenkov \& Denisenkova 1989; Langer et al. 1993; Arnould et al. 1999). In almost all GCs studied so far, ranging from the most massive one to the smallest objects in the Harris catalog (1996, and following updates), this pattern is retrieved; it is so typical a signature of GCs that a new definition of bona fide globular has been proposed based entirely on the presence of the most notable signature, the $\mathrm{Na}-\mathrm{O}$ anti-correlation (Carretta et al. 2010), discovered by the LickTexas group in the 1990s (see the review by Kraft 1994) and extensively studied in many clusters (see the updated review by Gratton et al. 2012, and references therein.).

While the first level of the game is clear, several details are still left in the poorly known age when GCs formed about a Hubble time ago. There are not enough data yet to uncover the true nature of the polluters that provided the material for building up the second generations. The most favourite candidates could be intermediate-mass asymptotic giant branch (AGB) stars (Ventura et al. 2001) and very massive, fast rotating stars (Decressin et al. 2007), although alternative ones have been presented, such as massive binaries (de Mink et al. 2009). Moreover, the distributions of stars along, e.g., the $\mathrm{Na}-\mathrm{O}$ anti-correlations do require that the matter composing the second stellar generation be diluted to some extent with material that did not experience hot nuclear burning. This rather strong constraint stems from both observations (the relations between proton-capture elements such as $\mathrm{O}$ and $\mathrm{Na}$ and the fragile element $\mathrm{Li}$, easily 
destroyed at temperatures much lower than those involved in hot H-burning, see Prantzos \& Charbonnel 2006 and the discussion in Gratton et al. 2012) and from theory (the yields from AGBs would necessarily result into a correlation, unless some amount of dilution is involved, D'Ercole et al. 2011).

Leaving aside for the moment several problems related to the nature of the diluting matter (D'Ercole et al. 2011), it is important to ascertain whether a model of dilution really does work and what the observational consequences are with respect to the formation of multiple stellar generations in GCs. Two scenarios can be envisioned:

1. After the formation of the first generation, a single mixture of matter from polluters diluted with unprocessed matter produces a second generation of stars distributed along the $\mathrm{Na}-\mathrm{O}$ and $\mathrm{Al}-\mathrm{Mg}$ anti-correlations. Since what is changing along these distributions is the degree of dilution, we expect that elements destroyed in the proton-capture reactions (namely, $\mathrm{O}$ and $\mathrm{Mg}$ ) or species enhanced in these processes (i.e., $\mathrm{Na}$ and $\mathrm{Al}$ ) will show a direct, linear proportionality.

2. On the other hand, several episodes of star formation might have occurred in the proto-GC after the formation of the first generation. Each one of them might have been characterized by a typical polluter with a given average mass. In this case, we expect that the elements are not necessarily produced or destroyed in linear proportion with each other. The distributions might still grossly resemble an anti-correlation, but different groups might be distinguished.

Ideally, we would like to have for these studies a database composed of many stars with detailed abundances of several light elements. This requires extensive sets of very high resolution and signal-to-noise (S/N) spectra with large spectral coverage, such as the FLAMES/UVES spectra obtained by Marino et al. (2008), who provided abundances for a good number of proton-capture elements $(\mathrm{O}, \mathrm{Na}, \mathrm{Mg}$, and $\mathrm{Al}$ for 93, 105, 105, and 87 stars, respectively) for M 4. When compared to our analysis for this cluster based on moderate-resolution GIRAFFE spectra (Carretta et al. 2009a), their analysis shows a clumpy distribution that was masked by noise on our lower quality data, despite the small starto-star errors derived by our tailored procedure. Unfortunately, it is not easy to obtain such high-quality material for a large number of clusters because such observations are very expensive in telescope time.

On the other hand, much insight can still be obtained by extending the spectral range of our moderate-resolution survey to other spectral ranges, allowing observations of transitions for elements whose data were missing in our original spectra. This can be obtained with very limited additional observing time exploiting the GIRAFFE spectrograph, which allows simultaneous observation of more than a hundred stars in a cluster, and can easily be done for several clusters. Using this technique, we showed, e.g., that the GC NGC 6752 is made of three discrete populations (Carretta et al. 2012a; we note that the presence of three groups of stars in NGC 6752 is quite clear, e.g., in the results obtained by Yong et al. 2008, but they did not make any comment about this in their paper). Moreover, we could show that the composition of the intermediate population of that cluster cannot be reproduced by simply mixing suitable amounts of the material which produced the two other populations. This clearly points towards the second scenario mentioned above, possibly supporting the finding by Yong et al. (2008) that contributions from both $\mathrm{AGB}$ and massive stars are required to explain the correlations in NGC 6752.
The present paper presents similar results on $\mathrm{Al}$ for two additional GCs (47 Tuc=NGC 104 and M 4=NGC 6121) for which we have already produced $\mathrm{Na}, \mathrm{O}$ abundances in our FLAMES survey. The analysis is similar to that presented in Carretta et al. (2012a) for NGC 6752 and in Carretta et al. (2012b) for NGC 1851.

While for M 4 our data largely overlap with those of Marino et al. (2008), for 47 Tuc this is the largest sample of red giant stars (RGB) with homogeneous $\mathrm{Na}, \mathrm{O}$, and $\mathrm{Al}$ abundances that we are aware of; the previous largest sample observed at medium-high resolution is represented by the 14 stars with UVES spectra analysed by our group in Carretta et al. (2009b) for $\mathrm{Al}$, and in Carretta et al. (2009a) for $\mathrm{Na}$ and $\mathrm{O}$ abundances.

\section{Observations and analysis}

The present work is based on two exposures, the first of $2700 \mathrm{~s}$ at airmass 1.487 (seeing $=1.23^{\prime \prime}$ ) acquired on 2010, July 30 for 47 Tuc and the second of $1600 \mathrm{~s}$ at airmass 1.240 (seeing=1.79") taken on 2010, April 14 with FLAMES@VLT-UT2 and the highresolution grating HR21. The resolution is 17300 at the center of the spectra, and the spectral range goes from about 8484 to about $9001 \AA$, including the $\mathrm{Al}$ I doublet at 8772-73 $\AA$, which is the main feature we were interested in. The positioning of fibers used the same configurations employed to observe the strong doublet of $\mathrm{Na}$ (at 5682 and $5688 \AA$ ) with the grating HR11 in Carretta et al. (2009a) to maximize the number of stars in the two clusters along the expected $\mathrm{Na}-\mathrm{Al}$ correlation. We observed 116 giants in 47 Tuc in the magnitude range $V=12.25-14.57$ and 83 RGB stars in the magnitude range $V=11.63-14.08$ in M 4. Coordinates and magnitudes for all stars in our samples can be retrieved in the online tables of Carretta et al. (2009a).

Data reduction was performed by the ESO personnel through the dedicated ESO FLAMES-GIRAFFE pipeline. The output bias-corrected, flat-fielded, one-dimensional, and wavelengthcalibrated spectra were sky subtracted and shifted to zero radial velocity using IRAF ${ }^{1}$.

The $\mathrm{S} / \mathrm{N}$ ratio of individual spectra is listed in Tables 2 and 3 (only available online) for 47 Tuc and M 4, respectively. The median $\mathrm{S} / \mathrm{N}$ is 249 for spectra of stars in 47 Tuc and 168 for giants observed in $\mathrm{M} 4$.

\section{Analysis and derived abundances}

The atmospheric parameters required for the analysis were simply adopted from Carretta et al. (2009a), where a full abundance analysis was done for all the stars targeted in the present study. This approach has two main advantages: first, it provides a full set of homogeneous parameters with very small star-to-star errors (typical internal errors are 4-6 $\mathrm{K}$ in temperature, $0.04 \mathrm{dex}$ in gravity, $0.03 \mathrm{dex}$ in $[\mathrm{Fe} / \mathrm{H}]$, and $0.02-0.03 \mathrm{~km} \mathrm{~s}^{-1}$ in microturbulent velocity, see Table A.2 in Carretta et al. 2009a), which is useful to minimize a possible smearing along the observed anti-correlations due only to errors associated to the analysis. Moreover, the number of lines of iron with well-known atomic parameters is not high in the spectral range of the grating HR21,

1 IRAF is the Image Reduction and Analysis Facility, a general purpose software system for the reduction and analysis of astronomical data. IRAF is written and supported by the IRAF programming group at the National Optical Astronomy Observatories (NOAO) in Tucson, Arizona. NOAO is operated by the Association of Universities for Research in Astronomy (AURA), Inc. under cooperative agreement with the National Science Foundation. 
Table 1. List of regions used to estimate local continuum and fluxes of $\mathrm{CN}$ bands.

\begin{tabular}{cccc}
\hline \hline & $\lambda_{\text {in }}$ & $\lambda_{\text {fin }}$ & $\Delta \lambda$ \\
& $\AA$ & $\AA$ & $\AA$ \\
\hline $\mathrm{CN}$ & 8759.22 & 8760.22 & 1.00 \\
$\mathrm{CN}$ & 8762.66 & 8763.40 & 0.74 \\
$\mathrm{CN}$ & 8767.24 & 8768.24 & 1.00 \\
$\mathrm{CN}$ & 8769.11 & 8769.92 & 0.81 \\
$\mathrm{CN}$ & 8769.96 & 8771.34 & 1.38 \\
$\mathrm{CN}$ & 8774.84 & 8776.12 & 1.28 \\
$\mathrm{CN}$ & 8782.88 & 8783.82 & 0.94 \\
$\mathrm{CN}$ & 8788.92 & 8789.61 & 0.69 \\
$\mathrm{CN}$ & 8794.02 & 8794.76 & 0.74 \\
$\mathrm{CN}$ & 8799.14 & 8800.06 & 0.92 \\
$\mathrm{CN}$ & 8801.77 & 8802.65 & 0.88 \\
$\mathrm{CN}$ & 8812.42 & 8813.42 & 1.00 \\
$\mathrm{CN}$ & 8813.42 & 8814.01 & 0.59 \\
$\mathrm{CN}$ & 8815.63 & 8816.52 & 0.89 \\
$\mathrm{CN}$ & 8819.04 & 8820.32 & 1.28 \\
$\mathrm{CN}$ & 8822.12 & 8823.38 & 1.26 \\
$\mathrm{CN}$ & 8830.32 & 8831.44 & 1.12 \\
$\mathrm{CN}$ & 8835.07 & 8836.86 & 1.79 \\
$\mathrm{cont}$ & 8739.29 & 8739.71 & 0.42 \\
cont & 8746.22 & 8746.53 & 0.31 \\
cont & 8765.41 & 8765.60 & 0.19 \\
cont & 8771.32 & 8771.65 & 0.33 \\
cont & 8791.26 & 8791.46 & 0.20 \\
cont & 8791.74 & 8792.12 & 0.38 \\
cont & 8794.76 & 8795.32 & 0.56 \\
cont & 8834.69 & 8835.14 & 0.45 \\
\hline & & &
\end{tabular}

whereas the $[\mathrm{Fe} / \mathrm{H}]^{2}$ values derived in Carretta et al. (2009a) rest on several iron lines. Second, $\mathrm{O}$ and $\mathrm{Na}$ abundances, homogeneously derived using the same atmospheric parameters, can be safely coupled with the new abundances of $\mathrm{Al}, \mathrm{Mg}$, and $\mathrm{Si}$.

The complete list of atmospheric parameters (effective temperature $T_{\text {eff }}$, surface gravity, metallicity, and microturbulent velocity) and the typical associated errors can be retrieved from the online tables in Carretta et al. (2009a) for all stars in 47 Tuc and M 4 analyzed in the present study.

\subsection{Nitrogen}

$\mathrm{CN}$ lines are ubiquitous in the observed spectral range, and some of them could also contaminate the $\mathrm{Al}$ lines. Thus, the first step is to reproduce the $\mathrm{CN}$ features as accurately as possible. The problem is more pronounced in metal-rich clusters such as 47 Tuc $([\mathrm{Fe} / \mathrm{H}]=-0.77$ dex, Carretta et al. 2009c), but some degree of contamination should also be expected in the more metal-poor cluster M 4 ([Fe/H] = -1.17 dex, Carretta et al. 2009c).

To deal with this aspect, we used an improved version of the procedure adopted in Carretta et al. (2012b). We first obtained a coadded spectrum with very high $\mathrm{S} / \mathrm{N}$ value by summing up the ten spectra of 47 Tuc stars with the highest $\mathrm{S} / \mathrm{N}$. On this master spectrum, we individuated eight regions apparently free of lines to be used to derive a local reference continuum and 18 spectral regions dominated by $\mathrm{CN}$ bands. Definitions of these spectral regions and of those for the local reference continuum are given in Table 1.

In the second step, synthetic spectra were computed using the Kurucz (1993) grid of model atmospheres (with the overshooting option switched on) and the same line list used in

\footnotetext{
2 We adopt the usual spectroscopic notation, i.e., for any given species $\mathrm{X},[\mathrm{X}]=\log \epsilon(\mathrm{X})_{\text {star }}-\log \epsilon(\mathrm{X})_{\odot}$ and $\log \epsilon(\mathrm{X})=\log \left(N_{\mathrm{X}} / N_{\mathrm{H}}\right)+12.0$ for absolute number density abundances.
}

Carretta et al. (2012b), adopting the atmospheric parameters suitable for each individual star in 47 Tuc. Three spectra were computed assuming $[\mathrm{C} / \mathrm{Fe}]=-0.6 \mathrm{dex}$ and three evenly spaced values of nitrogen, $[\mathrm{N} / \mathrm{Fe}]=-0.5,+0.25$ and $+1.0 \mathrm{dex}$, with the package ROSA (Gratton 1988). We applied the procedure explained in Carretta et al. (2012b) to each of the $18 \mathrm{CN}$ features in each star: the average flux within the in-line region of the feature was measured and a weighted reference continuum was derived from all the eight off-line regions using weights equal to the width of each region. Whenever a defect in the spectrum was found on a continuum region from visual inspection, that region was excluded from the computation of the reference local continuum. The $N$ abundance from each feature was then obtained by comparing the normalized flux with the fluxes measured in the same way on the three synthetic spectra.

The above procedure was repeated for each of the 116 stars observed in 47 Tuc, and for each star an average [N/Fe] value was derived. The spectral regions of each $\mathrm{CN}$ feature were inspected visually and the relative feature discarded from the average whenever a spike was found to fall on the feature. The final abundances of $\mathrm{N}$ were obtained after applying a $\mathrm{k} \sigma$-clipping at $2.5 \sigma$ to the average abundance from individual features in each star. These final abundances in 47 Tuc rest on average on 17 features. We found a mean value $[\mathrm{N} / \mathrm{Fe}]=+1.038 \pm 0.011$ $\operatorname{dex}(\mathrm{rms}=0.119$ dex, 116 stars).

This approach was also adopted for the 83 stars observed in $\mathrm{M} \mathrm{4}$; also in this case, the mean $\mathrm{N}$ abundance was derived from an average of $17 \mathrm{CN}$ features, finding $[\mathrm{N} / \mathrm{Fe}]=$ $+1.052 \pm 0.017 \mathrm{dex}(\mathrm{rms}=0.152 \mathrm{dex}, 83 \mathrm{stars})$. The larger scatter observed in M 4 is mainly due to the giants in this more metal-poor cluster being on average $200 \mathrm{~K}$ warmer than giants in 47 Tuc (and only minimally due to the lower $\mathrm{S} / \mathrm{N}$ of the spectra). The molecular features are more difficult to measure, and the warmest stars are thus responsible for most of the observed scatter.

Individual values of $\mathrm{N}$ abundances are listed in Tables 2 and 3 for 47 Tuc and $\mathrm{M} \mathrm{4,} \mathrm{respectively.} \mathrm{We} \mathrm{recall} \mathrm{that} \mathrm{these}$ $[\mathrm{N} / \mathrm{Fe}]$ were obtained assuming $[\mathrm{C} / \mathrm{Fe}]=-0.6$. Were the $\mathrm{C}$ abundance for a given star different from this value, $[\mathrm{N} / \mathrm{Fe}]$ should be modified in such a way that the sum $[\mathrm{C} / \mathrm{Fe}]+[\mathrm{N} / \mathrm{Fe}]$ remains constant, because the concentration of $\mathrm{CN}$ depends on the product of the $\mathrm{C}$ and $\mathrm{N}$ concentrations.

The $\mathrm{C}$ abundances are expected to change as a function of temperature due to deep mixing. We estimated from $\mathrm{C}$ and $\mathrm{N}$ abundances measured in red giants of $\mathrm{M} 4$ by Ivans et al. (1999) that over the temperature range of our sample the maximum variation in $\mathrm{C}$ expected as a function of the luminosity is about 0.25 dex. The effect of star-to-star variations in $\mathrm{C}$ abundances at a given luminosity, related to the multiple population phenomenon, will be discussed later. The impact is likely very important in the case of 47 Tuc. For M 4, it seems less of a problem. For instance, we have sixteen stars in common with Smith \& Briley (2005) who obtained C abundances from lowresolution spectra. If we divide these stars in six Na-poor and ten Na-rich stars $($ at $[\mathrm{Na} / \mathrm{Fe}]=0.4)$, we find $[\mathrm{C} / \mathrm{Fe}]=-0.57 \pm 0.04$ $(\mathrm{rms}=0.10)$ and $[\mathrm{C} / \mathrm{Fe}]=-0.64 \pm 0.06 \mathrm{dex}(\mathrm{rms}=0.20 \mathrm{dex})$ for the two groups, both compatible with our assumption of $[\mathrm{C} / \mathrm{Fe}]=-0.6$.

We tested the adopted procedure on the spectrum of Arcturus $^{3}$. We obtained $[\mathrm{N} / \mathrm{Fe}]=0.68$ adopting $[\mathrm{C} / \mathrm{Fe}]=0$

We retrieved this spectrum in the relevant wavelength range from the ESO Advanced Data Product Archive

(http://archive.eso.org/eso/eso_archive_adp.html). 
Table 4. Sensitivities of $\mathrm{Al}$ to variations in the atmospheric parameters and errors in fluxes, and errors in abundances [A/Fe] for stars in $47 \mathrm{Tuc}$ and $\mathrm{M} 4$.

\begin{tabular}{lrrrrrrr}
\hline \hline 47 Tuc & & & & & & & \\
\hline Element & {$[\mathrm{N} / \mathrm{Fe}]$} & $\begin{array}{r}T_{\text {eff }} \\
(\mathrm{K})\end{array}$ & $\begin{array}{r}\log g \\
(\mathrm{dex})\end{array}$ & $\begin{array}{r}{[\mathrm{A} / \mathrm{H}]} \\
(\mathrm{dex})\end{array}$ & $\begin{array}{r}v_{\mathrm{t}} \\
\mathrm{km} \mathrm{s}^{-1}\end{array}$ & $\begin{array}{r}\text { Flux } \\
(\mathrm{dex})\end{array}$ & $\begin{array}{r}\text { Total } \\
\text { Internal }\end{array}$ \\
\hline Variation & +0.200 & 50 & 0.200 & 0.100 & 0.10 & & \\
Internal & +0.012 & 6 & 0.020 & 0.032 & 0.11 & 0.013 & \\
\hline$[\mathrm{Al} / \mathrm{Fe}] \mathrm{I}$ & 0.077 & -0.032 & 0.000 & 0.115 & +0.013 & & 0.042 \\
\hline
\end{tabular}

\begin{tabular}{lrrrrrrr}
\hline \hline M 4 & & & & & & & \\
\hline Element & {$[\mathrm{N} / \mathrm{Fe}]$} & $\begin{array}{r}T_{\text {eff }} \\
(\mathrm{K})\end{array}$ & $\begin{array}{r}\log g \\
(\mathrm{dex})\end{array}$ & $\begin{array}{r}{[\mathrm{A} / \mathrm{H}]} \\
(\mathrm{dex})\end{array}$ & $\begin{array}{r}v_{\mathrm{t}} \\
\mathrm{km} \mathrm{s}^{-1}\end{array}$ & $\begin{array}{r}\text { Flux } \\
(\mathrm{dex})\end{array}$ & $\begin{array}{r}\text { Total } \\
\text { Internal }\end{array}$ \\
\hline Variation & +0.200 & 50 & 0.200 & 0.100 & 0.10 & & \\
Internal & +0.028 & 4 & 0.041 & 0.025 & 0.12 & 0.019 & \\
\hline$[\mathrm{Al} / \mathrm{Fe}] \mathrm{I}$ & 0.013 & -0.031 & 0.030 & 0.104 & +0.012 & & 0.036 \\
\hline
\end{tabular}
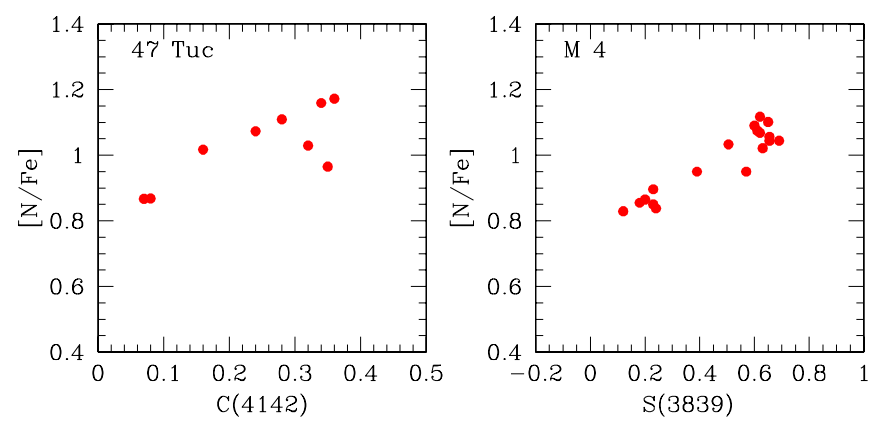

Fig. 1. Left: comparison of our derived $[\mathrm{N} / \mathrm{Fe}]$ ratios with the values of the DDO index from Norris \& Freeman (Norris1979b) in 47 Tuc. Right: comparison of our $N$ abundances with the $\mathrm{S}(3839)$ index for the strength of the $\lambda 3883 \mathrm{CN}$ band from the compilation by Smith \& Briley (2005) in $\mathrm{M} 4$.

and the atmospheric parameters from Hinkle et al. (2000) and Carlberg et al. (2012): $T_{\text {eff }}=4300 \mathrm{~K}, \log g=1.70,[\mathrm{Fe} / \mathrm{H}]=$ -0.55 , and $\left.v_{\mathrm{t}}=1.64\right)$. This result is very similar to that obtained by D'Orazi et al. (2011) with very similar atmospheric parameters.

For a check, we searched the literature for analysis with published values of $\mathrm{N}$ or $\mathrm{CN}$ (or related indexes) in these two clusters. We found $11 \mathrm{RGB}$ stars of 47 Tuc in common with the sample studied by Norris \& Freeman (1979), and we measured $N$ abundances for 9 of them; we also have 29 stars in common with the compilation of $\mathrm{CN}$ values by Smith \& Briley (2005) in M 4, with our $N$ values available for 18 of them. In Fig. 1 we show the comparison of our derived $N$ abundances with the values of the DDO index C(4142) in 47 Tuc (left-hand panel) and of the re-homogenized index S(3839) by Smith \& Briley (2005) in M 4 (right-hand panel). In both cases, with our assumptions about the carbon abundance, our values are quite well correlated with these literature values. We conclude that our values provide a good estimate of the $\mathrm{N}$ content in the program stars.

\subsection{Aluminium, magnesium, and silicon}

A similar procedure was used to derive $\mathrm{Al}$ abundances (see also Carretta et al. 2012b). We used for each star three synthetic spectra computed with the appropriate atmospheric parameters (from Carretta et al. 2009a) and the $N$ abundances derived as described above. The fluxes for determining $\mathrm{Al}$ were measured in the in-line region from 8772.4 to $8774.7 \AA$ while two slightly different regions were used in the two clusters to derive the local reference continuum owing to the different metallicity of these GCs. The selected regions were 8771.28-8771.70 $\AA$ and 8776.54-8777.46 $\AA$ in 47 Tuc and 8768.90-8770.00 $\AA$ and 8775.20-8776.10 $\AA$ in M 4. Again, we obtained a final continuum by averaging the contribution from the two regions with weights given by the number of pixels (i.e., the widths of these intervals).

Abundances of $\mathrm{Al}$ were then derived by interpolating the normalized fluxes in the in-line $\mathrm{Al}$ region among those obtained from the synthetic spectra, computed for $[\mathrm{Al} / \mathrm{Fe}]=0.0,0.75$, 1.5 dex. As above, should one of the reference local continuum regions or one of the $\mathrm{Al}$ lines be affected by a defect, the contaminated region would be dropped from the computation, following visual inspection.

Abundances of $\mathrm{Mg}$ and $\mathrm{Si}$ were obtained from the same set of lines described in Carretta et al. (2012a), using atmospheric parameters derived in Carretta et al. (2009a). Final $\mathrm{Al}, \mathrm{Mg}$, and Si abundances for giants in 47 Tuc and M 4 are listed in Tables 2 and 3 , respectively.

\subsection{Errors}

Star-to-star errors in the adopted atmospheric parameters were estimated in Carretta et al. (2009a) and are shown in Table 4. To translate these errors into errors on the $\mathrm{Al}$ abundances, we need the sensitivities of Al to change in the adopted parameters. These were obtained by repeating the analysis for the stars 29490 $\left(T_{\text {eff }}=4584 \mathrm{~K}\right)$ in 47 Tuc and star $30450\left(T_{\text {eff }}=4622 \mathrm{~K}\right)$ in M 4 (these stars were chosen because they have an effective temperature in the middle of the temperature range of their respective samples) and changing a parameter each time, holding the others fixed at their original value. The amount of variations and the sensitivity of each parameter to these changes are listed in Table 4.

The star-to-star (internal) errors in the $\mathrm{Al}$ abundances are obtained by summing in quadrature all the contributions, including the impact of errors in the derivation of $N$ abundances from $\mathrm{CN}$ features.

We attached an error due to flux measurement to the $\mathrm{Al}$ abundances. As in Carretta et al. (2012b), this was evaluated by estimating photometric errors from the $\mathrm{S} / \mathrm{N}$ of the spectra of stars and from the width within each of the reference continuum and in-line regions (and then the number of independent pixels used). The error in $\mathrm{Al}$ abundances is then obtained by comparing these 

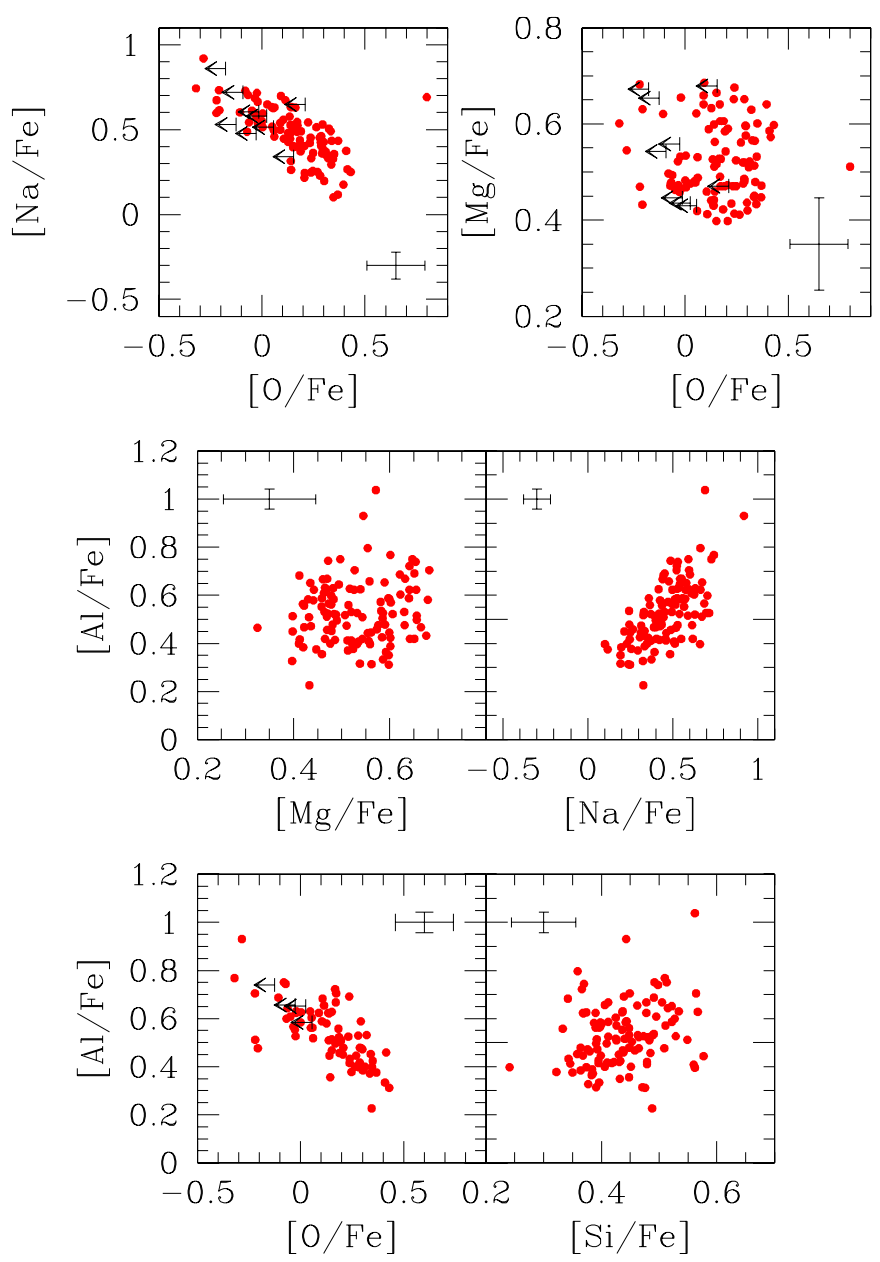

Fig. 2. Run of the abundance ratios of proton-capture elements $\mathrm{O}, \mathrm{Na}$, $\mathrm{Mg}, \mathrm{Al}$, and Si among red giants in 47 Tuc from Carretta et al. (2009a) and the present study. Upper limits in $\mathrm{O}$ are indicated by arrows. Typical star-to-star errors are also indicated.

Al abundances with those derived considering a new value of the Al line strength index that is the sum of the original values and its error. The errors from these procedure are listed for each star in Table 2 and 3; on average, they are $+0.013 \pm 0.001$ dex from 116 stars in 47 Tuc and $+0.019 \pm 0.001$ dex from 83 giants in $\mathrm{M} 4$.

Moreover, we applied the same approach to one of the $\mathrm{CN}$ features used to estimate the $N$ abundance. The average error is 0.012 dex in 47 Tuc and +0.028 dex in M 4. These values were assumed as conservative estimates in the error budget, since final $N$ abundances were obtained from a large number of features.

Summing up all the above contributions, the typical internal errors in the derived $\mathrm{Al}$ abundances are 0.042 dex and 0.036 dex for 47 Tuc and $M 4$, respectively. Typical internal errors in $[\mathrm{Mg} / \mathrm{Fe}]$ and $[\mathrm{Si} / \mathrm{Fe}]$, estimated according to the usual procedure (Carretta et al. 2009a) are 0.10 dex and 0.06 dex, respectively, for both clusters.

\section{Results}

The pattern of abundances of proton-capture elements among red giants in 47 Tuc is summarized in Figs. 2 and 3.

The Na-O anti-correlation in 47 Tuc was already known to be rather short, when considering its large total mass, which
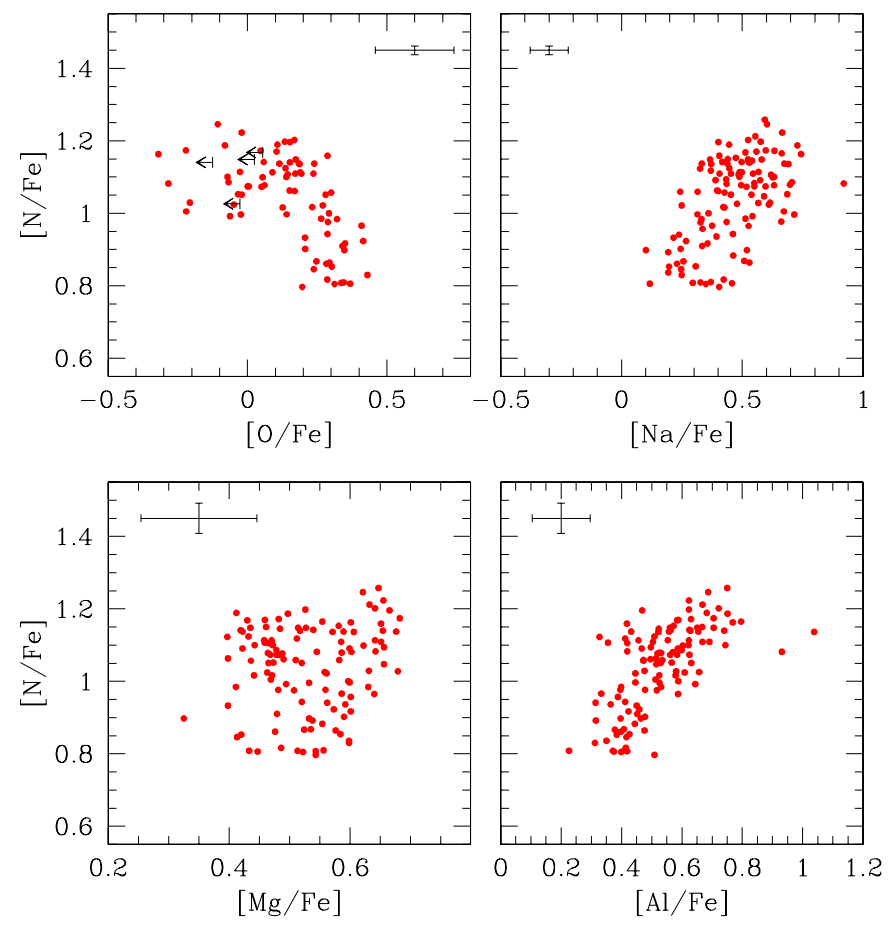

Fig. 3. Run of the nitrogen abundances as a function of $\mathrm{O}, \mathrm{Na}, \mathrm{Mg}$, and Al for our sample of RGB stars in 47 Tuc.

is one of the main parameters driving the extent of the anticorrelation (Carretta et al. 2010). The new set of light elements added in the present work confirms this trend: $\mathrm{Mg}$ and $\mathrm{Si}$ do not show large star-to-star variations, the average values being $[\mathrm{Mg} / \mathrm{Fe}]=+0.532 \pm 0.007 \mathrm{dex}(\mathrm{rms}=0.079,147$ stars $)$ and $[\mathrm{Si} / \mathrm{Fe}]=+0.440 \pm 0.005 \mathrm{dex}(\mathrm{rms}=0.065,147$ stars. The number of stars where $\mathrm{Mg}$ and $\mathrm{Si}$ are measured is higher than those with $\mathrm{Al}$ abundances since transitions of $\mathrm{Mg}$ and $\mathrm{Si}$ are available in the spectral range of both the gratings HR11 and HR13, whereas Al from GIRAFFE spectra is available for only the subsample observed with the grating HR21. The aluminium abundances span a range of about 0.5 dex among giants in this cluster, with an average value of $[\mathrm{Al} / \mathrm{Fe}]=+0.529 \pm 0.012 \mathrm{dex}$ $(\mathrm{rms}=0.132,116$ stars $)$. Clear trends of $\mathrm{Al}$ ratios correlated to $\mathrm{Na}$ and anti-correlated to $\mathrm{O}$ abundances show the presence in second-generation stars of ejecta processed through the hightemperature $\mathrm{Mg}-\mathrm{Al}$ cycle. This finding is also confirmed by the correlation (shallow, but still statistically robust) between $\mathrm{Si}$ and $\mathrm{Al}$, which by itself points toward a temperature of $\mathrm{H}$-burning in excess of $\sim 65 \times 10^{6} \mathrm{~K}$, where the leakage from the $\mathrm{Mg}$-Al cycle on ${ }^{28} \mathrm{Si}$ is onset (see Yong et al. 2005; Carretta et al. 2009b).

As expected, the abundances of $\mathrm{N}$ derived from the transformation of $\mathrm{O}$ in the $\mathrm{CNO}$ cycle are nicely correlated with elements enhanced in proton-capture reactions (such as $\mathrm{Na}, \mathrm{Al}$ ) and anti-correlated with oxygen (Fig. 3). The apparent lack of a N-Mg anti-correlation is not a large source of concern, since the $\mathrm{Mg}$ variations are small. Based on limited samples of RGB stars observed with FLAMES-UVES (Carretta et al. 2009b), our survey of $\mathrm{Al}$ and $\mathrm{Mg}$ abundances in several GCs shows that Al-rich and $\mathrm{Mg}$-depleted stars are present only in massive (NGC 2808, NGC 6388, NGC 6441) or metal-poor (NGC 6752) clusters, or both (NGC $7078=$ M 15). In such clusters, a clear $\mathrm{Mg}-\mathrm{Al}$ anticorrelation is observed even among main sequence stars (see, e.g., Bragaglia et al. 2010, for NGC 2808). In GCs such as those under scrutiny, small and/or metal-rich, large starto-star variations of $\mathrm{Mg}$ abundances are not expected, as shown 

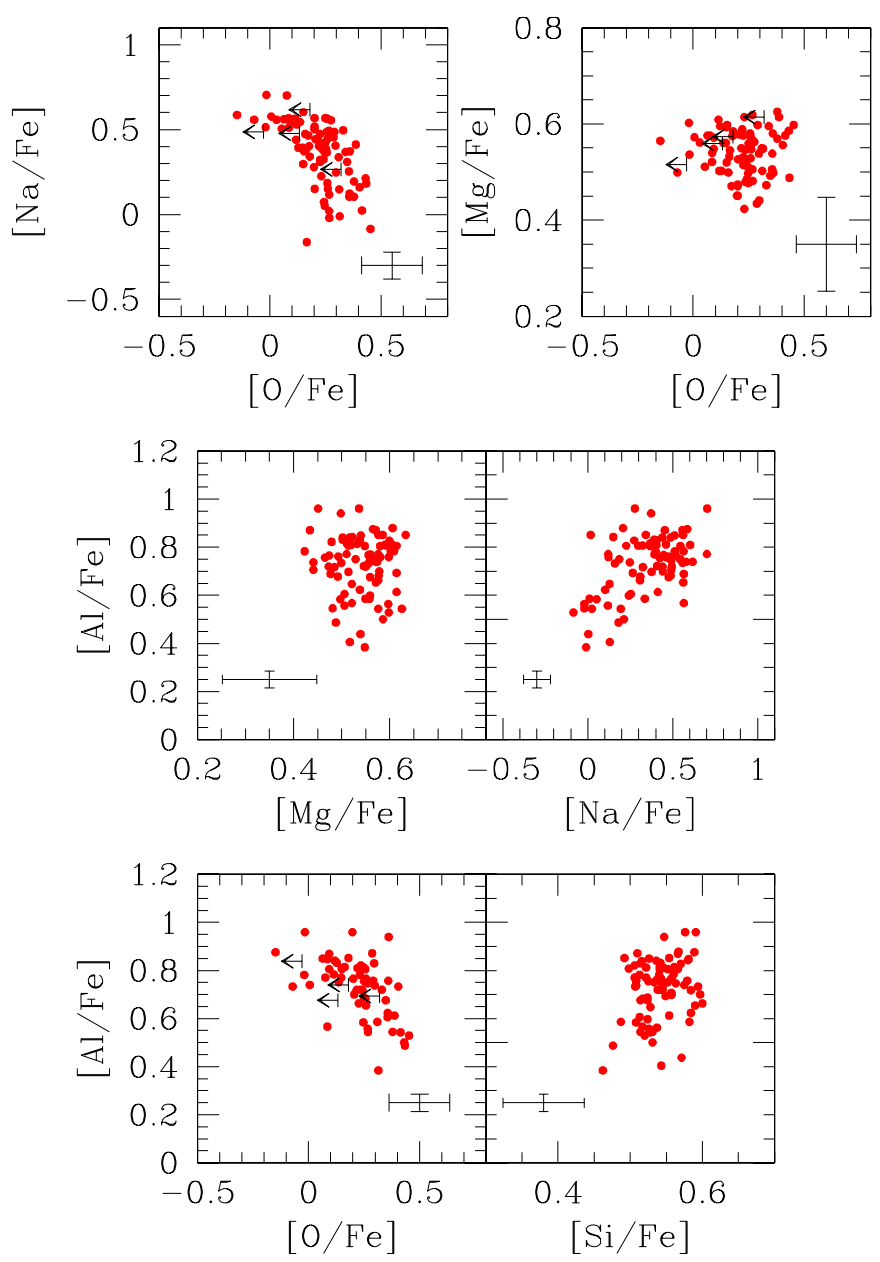

Fig. 4. The same as in Fig. 2 for M 4.

by Carretta et al. (2009b) in a survey of limited samples of giants in 15 GCs and by Marino et al. (2008) in about 100 giants in M 4.

The analogous run of light elements in the globular cluster M 4 is shown in Figs. 4 and 5. In this small mass cluster, the Na-O anti-correlation is found to be of moderate extension (Carretta et al. 2009a; Marino et al. 2008; Ivans et al. 1999), and also star-to-star variations in $\mathrm{Mg}$ and $\mathrm{Si}$ are not large. The average values from our analysis are $[\mathrm{Mg} / \mathrm{Fe}]=+0.541 \pm 0.005 \mathrm{dex}$ $(\mathrm{rms}=0.048,103$ stars $)$ and $[\mathrm{Si} / \mathrm{Fe}]=+0.540 \pm 0.003 \mathrm{dex}$ ( $\mathrm{rms}=0.034,103$ stars $)$. As already indicated by previous analyses (Marino et al. 2008; Carretta et al. 2009b), star-to-star variations in $\mathrm{Al}$ abundances are not large along the RGB in M 4. A clear $\mathrm{Na}-\mathrm{Al}$ correlation is evident in our data and in the UVES data of the large sample in Marino et al. (2008), whereas it cannot be seen in the limited sample of stars analyzed in Carretta et al. (2009b). On the other hand, no correlation between Si and $\mathrm{Al}$ can be seen in the present study.

Concerning $\mathrm{N}$ abundances, we found a somewhat larger scatter with respect to the results of 47 Tuc. As explained above, this is largely due to the increased difficulty in measuring $\mathrm{CN}$ features in the warmest stars of our sample in this more metal-poor cluster. This is shown in Fig. 6, where [N/Fe] ratios are plotted as a function of the effective temperature. The scatter noticeably increases for stars at the warmest end of the sample, and these stars account for most of the dispersion seen in Fig. 5.
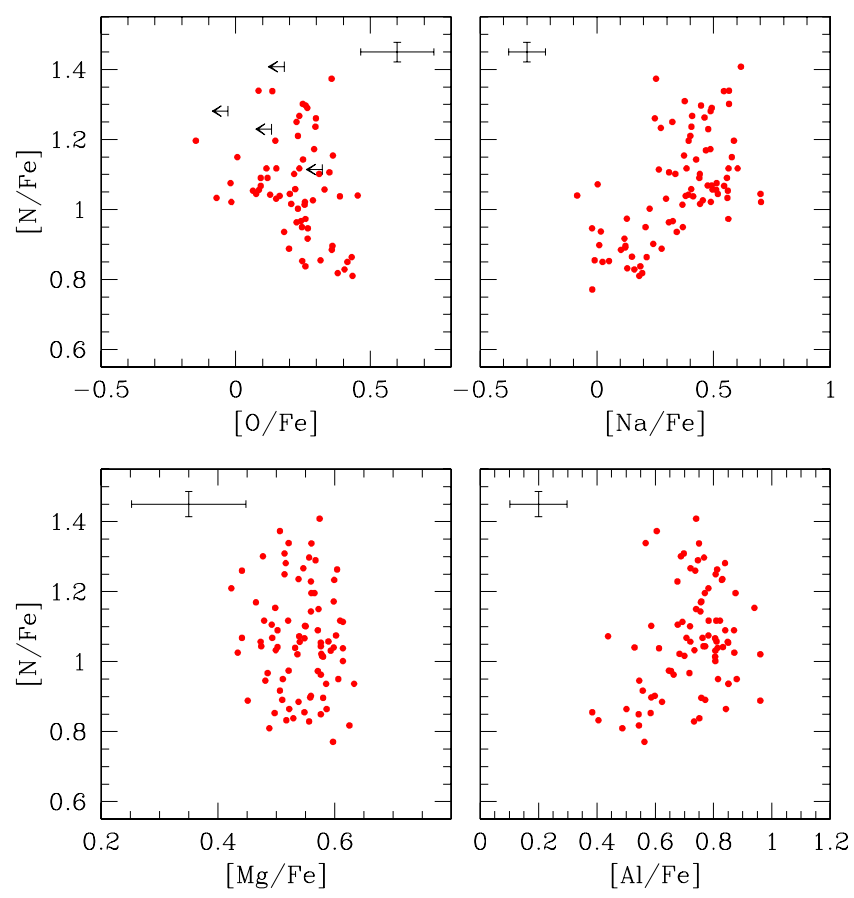

Fig. 5. The same as in Fig. 3 for M 4.

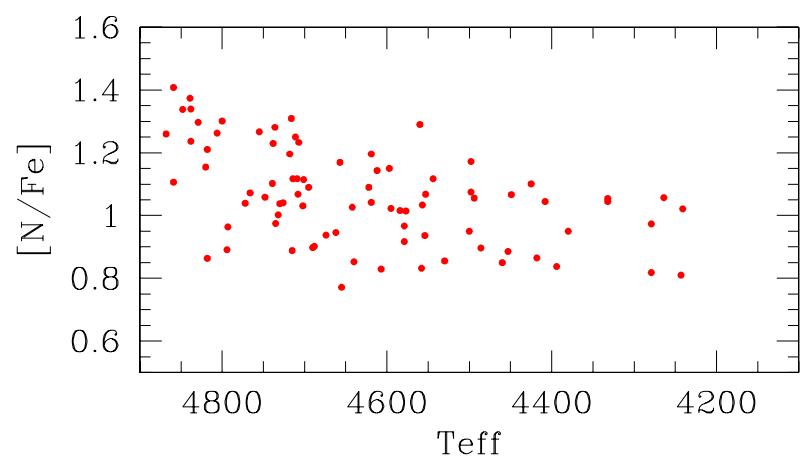

Fig. 6. Abundances of $\mathrm{N}$ as a function of effective temperature in giants of M 4 .

\subsection{Comparison with previous studies}

We found in our sample 85 stars in common with the analysis of M 4 by Marino et al. (2008); the comparison between our abundances of $\mathrm{Na}, \mathrm{Al}, \mathrm{Mg}$, and $\mathrm{Si}$ is summarized in Fig. 7.

Taking into account differences in the scale of atmospheric parameters (based on photometry in our case; eventually adjusted using spectroscopic parameters in the analysis made by M08), abundance indicators (M08 used the classical optical doublet of Al I at 6696-98 $\AA$ instead of the stronger doublet at $8772-73 \AA$ as in the present work), atomic parameters, and the different resolution of the spectra (higher resolution UVES spectra for M08), the agreement is satisfactory.

Concerning 47 Tuc, recently Gratton et al. (2013) studied a sample of 110 stars on the red horizontal branch (HB) in 47 Tuc. The agreement in $\mathrm{O}$ and $\mathrm{Na}$ abundances is very good: the Na-O anti-correlation as observed along the RGB and the $\mathrm{HB}$ is almost indistinguishable (see Fig. 5 in Gratton et al.), despite the different abundance indicators adopted in the two studies. However, for $\mathrm{N}$ we found that an offset exists, the bulk of $[\mathrm{N} / \mathrm{Fe}]$ values being found between $\sim 1.2$ and $\sim 2$ dex for HB stars in 47 Tuc. Finally, some offset is also seen regarding 

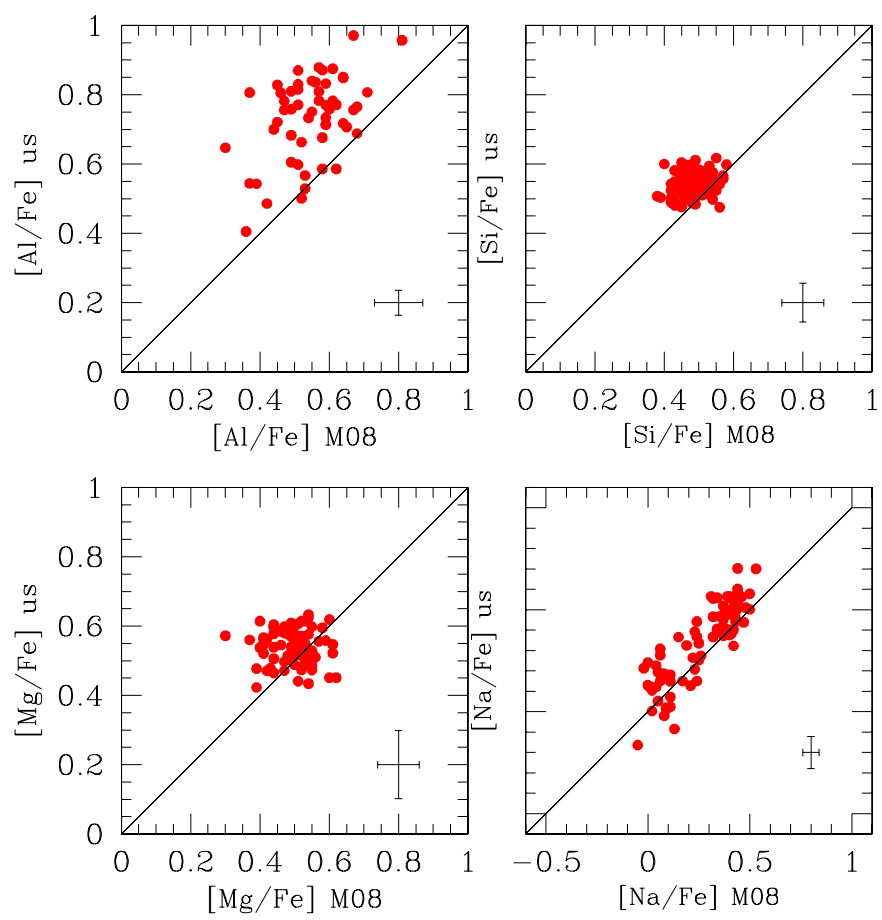

Fig. 7. Comparison of abundance ratios of $\mathrm{Na}, \mathrm{Al}, \mathrm{Mg}$, and $\mathrm{Si}$ for stars in our sample in common with the study by Marino et al. (2008). The solid line is the line of equality.

$\mathrm{Al}$ abundances (Fig. 14 in Gratton et al.), which are derived from different doublets (7835-36 ̊ for HB stars and 8772-83 ̊ here).

\subsection{Cluster analysis}

The large dataset (both in number of elements and in number of stars) available to us allowed us to perform a cluster analysis to find out whether stars observed along the relations among light elements form discrete groups. As in Carretta et al. (2012a), this was done using the $k$-means algorithm (Steinhaus 1956; MacQueen 1967) as implemented in the $R$ statistical package (R Development Core Team 2011; http://www.R-project. org). For this exercise we selected the abundance ratios $[\mathrm{Al} / \mathrm{Fe}]$, $[\mathrm{O} / \mathrm{Fe}],[\mathrm{Na} / \mathrm{Fe}],[\mathrm{Mg} / \mathrm{Fe}]$, and $[\mathrm{N} / \mathrm{Fe}]$, considering only stars in 47 Tuc and M 4 with all these elements simultaneously determined: 79 giants in 47 Tuc and 67 in M 4. The parameters were weighted according to their internal errors, which are largest for $\mathrm{O}$ abundances, usually derived by only one line (the associated internal errors in $\mathrm{O}$ are, for example, about twice those relative to $\mathrm{Na}$; see the appendix in Carretta et al. 2009a).

The number of subgroups to be found is a parameter that must be provided as input to the cluster analysis when using the $k$-means algorithm. The simplest hypothesis we could make is that two groups, i.e., stellar generations, exist in the cluster. The first one has a primordial, typical supernovae-enriched abundance pattern; the second has a chemical composition modified by ejecta of a fraction of stars of the first generation (the polluters).

The results are shown in the upper panel of Fig. 8 for stars in 47 Tuc; we used as $x$-axis a parameter including all the elements that are enhanced in the proton-capture reactions. This parameter quantifies how much the second generation is extreme. We display the ratio $[\mathrm{O} / \mathrm{Fe}]$ in ordinate, since it is the element most susceptible to depletion through these reactions, while Mg variations are, for example, usually very tiny. The average abundances

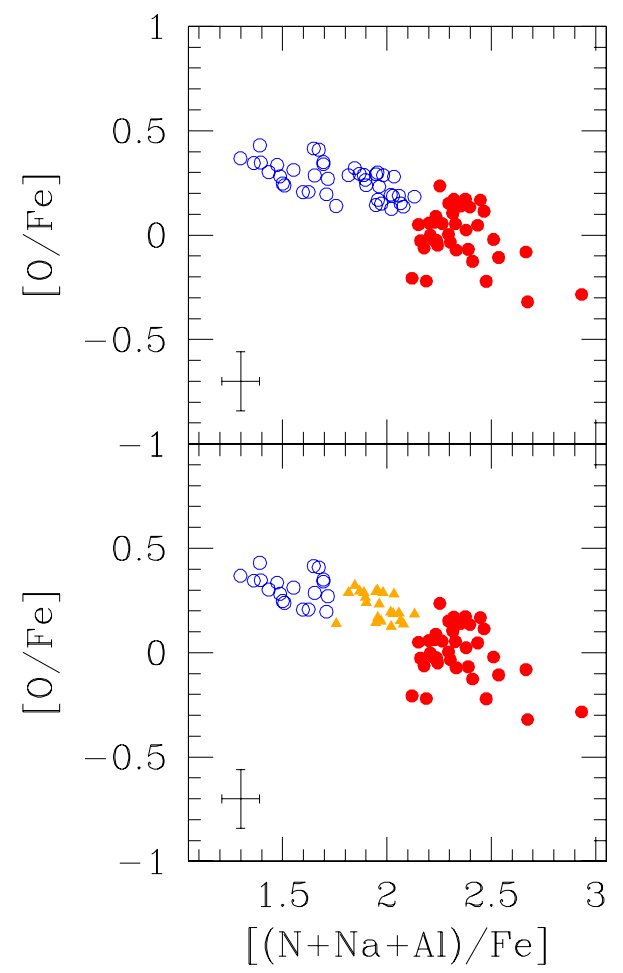

Fig. 8. Upper panel: results of the cluster analysis on stars in 47 Tuc with a full set of abundances of $\mathrm{N}, \mathrm{O}, \mathrm{Na}, \mathrm{Mg}, \mathrm{Al}$. The input number of groups to be found is two. Lower panel: the same, but using three as the number of groups in input.

for each group are listed in Table 5. The algorithm basically finds in 47 Tuc two groups that correspond to the two generations, assigning about half of the stars to each group. A fraction of $50 \%$ of stars of the first generation is somewhat higher than the fraction of $\sim 30 \%$, according to the definition of primordial $\mathrm{P}$ fraction given in Carretta et al. (2009a).

However, looking at the upper panel in Fig. 8, we note that beside the rather clear separation between stars in the first (open circles) and in the second groups (filled circles) there is some evidence for discontinuity in the distribution within the first group, located approximatively at $[(\mathrm{N}+\mathrm{Na}+\mathrm{Al}) / \mathrm{Fe}]=1.8 \mathrm{dex}$. To verify how sound this feature is, we ran the cluster analysis again, forcing the algorithm to find three groups.

The results are presented in the lower panel of Fig. 8. The stars of the previous first generation are now split into two groups and the putative intermediate population detected above nicely falls into a unique group (filled triangles), apart from a single outlier. With this separation, the fraction of stars with primordial composition is now $24 \%$, while the intermediate and extreme populations summed together reach $76 \%$ of the sample, reassuringly close to the typical fractions derived in Carretta et al. (2009a) using only $\mathrm{O}$ and $\mathrm{Na}$ abundances. This is not surprising, since the positioning of the GIRAFFE fibers with the HR21 grating in the present study was done with the same configuration used for $\mathrm{Na}$ (HR11) by Carretta et al. (2009a). As explained in that paper, these configurations, due to the limitations of the Oz-Poz positioner of FLAMES, are not the best suited to study the radial dependence of the fraction of stars in the P, I, and $\mathrm{E}$ components. This also makes difficult a direct comparison with methods based on photometric parameters (like those in Milone et al. 2012), which are better able to study radial distributions, but with different sensitivities to chemistry and age. For instance, we note that in Milone et al. three populations were 
Table 5. Average values of abundance ratios of the different groups resulting from the cluster analysis in 47 Tuc and M 4.

\begin{tabular}{|c|c|c|c|c|c|c|}
\hline \multicolumn{7}{|c|}{47 Tuc: 2 groups } \\
\hline group & $\mathrm{nr}$ & {$[\mathrm{N} / \mathrm{Fe}]$} & {$[\mathrm{O} / \mathrm{Fe}]$} & {$[\mathrm{Na} / \mathrm{Fe}]$} & {$[\mathrm{Mg} / \mathrm{Fe}]$} & {$[\mathrm{Al} / \mathrm{Fe}]$} \\
\hline 1 & 41 & $+0.98 \pm 0.02$ & $+0.26 \pm 0.01$ & $+0.36 \pm 0.02$ & $+0.52 \pm 0.01$ & $+0.45 \pm 0.01$ \\
\hline 2 & 38 & $+1.11 \pm 0.01$ & $+0.00 \pm 0.02$ & $+0.59 \pm 0.02$ & $+0.52 \pm 0.01$ & $+0.64 \pm 0.01$ \\
\hline \multicolumn{7}{|c|}{47 Tuc: 3 groups } \\
\hline 1 & 19 & $+0.87 \pm 0.01$ & $+0.31 \pm 0.02$ & $+0.28 \pm 0.02$ & $+0.51 \pm 0.02$ & $+0.40 \pm 0.01$ \\
\hline 2 & 22 & $+1.06 \pm 0.02$ & $+0.22 \pm 0.02$ & $+0.42 \pm 0.01$ & $+0.52 \pm 0.02$ & $+0.49 \pm 0.01$ \\
\hline 3 & 38 & $+1.11 \pm 0.02$ & $+0.00 \pm 0.01$ & $+0.59 \pm 0.02$ & $+0.52 \pm 0.01$ & $+0.64 \pm 0.01$ \\
\hline
\end{tabular}

\begin{tabular}{|c|c|c|c|c|c|c|}
\hline \multicolumn{7}{|c|}{ M 4: 2 groups } \\
\hline group & $\mathrm{nr}$ & {$[\mathrm{N} / \mathrm{Fe}]$} & {$[\mathrm{O} / \mathrm{Fe}]$} & {$[\mathrm{Na} / \mathrm{Fe}]$} & {$[\mathrm{Mg} / \mathrm{Fe}]$} & {$[\mathrm{Al} / \mathrm{Fe}]$} \\
\hline 1 & 13 & $+0.88 \pm 0.01$ & $+0.35 \pm 0.02$ & $+0.10 \pm 0.02$ & $+0.55 \pm 0.01$ & $+0.58 \pm 0.03$ \\
\hline 2 & 54 & $+1.12 \pm 0.03$ & $+0.19 \pm 0.03$ & $+0.45 \pm 0.03$ & $+0.54 \pm 0.01$ & $+0.77 \pm 0.02$ \\
\hline \multicolumn{7}{|c|}{ M 4: 3 groups } \\
\hline 1 & 13 & $+0.88 \pm 0.01$ & $+0.35 \pm 0.02$ & $+0.10 \pm 0.02$ & $+0.55 \pm 0.01$ & $+0.58 \pm 0.03$ \\
\hline 2 & 24 & $+1.21 \pm 0.01$ & $+0.26 \pm 0.02$ & $+0.42 \pm 0.02$ & $+0.53 \pm 0.01$ & $+0.73 \pm 0.03$ \\
\hline 3 & 30 & $+1.05 \pm 0.02$ & $+0.13 \pm 0.03$ & $+0.47 \pm 0.03$ & $+0.55 \pm 0.01$ & $+0.80 \pm 0.02$ \\
\hline
\end{tabular}

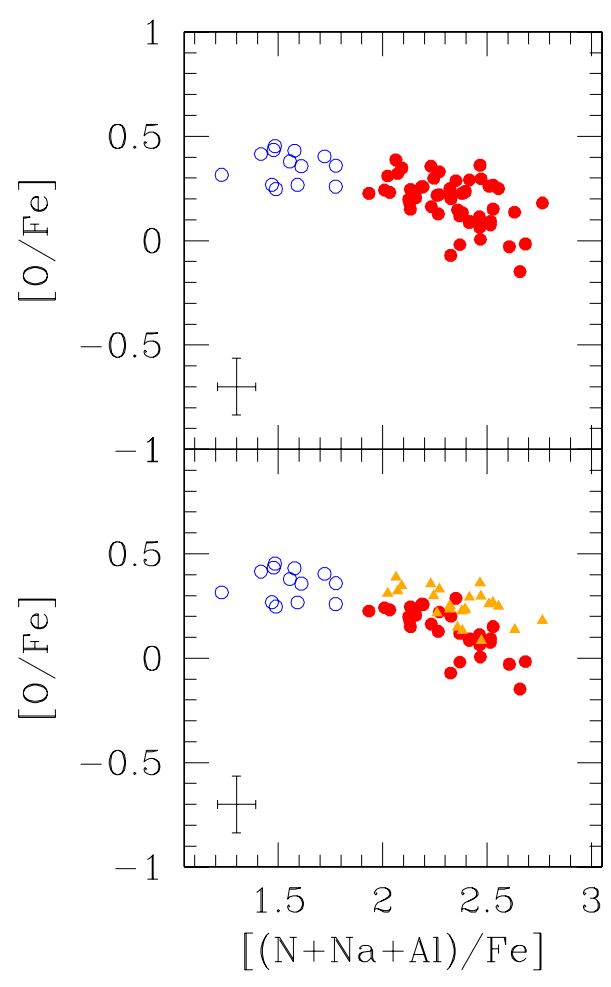

Fig. 9. The same as in Fig. 8 for M 4 .

found among subgiants, but not among RGB stars. Also, we are not sure that the three populations found along the RGB in the present study by means of chemical composition are the same as those found along the sub giant branch (SGB).

For M 4 the case is much simpler. The cluster analysis with only two groups as input separates quite well the first- and second-generation stars, as evident in the upper panel of Fig. 9, where the gap along the "secondness" parameter is very clear. Hence, it is not surprising that the cluster analysis fails to find three distinct subgroups (lower panel in Fig. 9). In this case, an "intermediate" group is found by the algorithm, but it is barely separated from the bulk of second-generation stars. A look at the abundances listed in Table 5 shows that this putative intermediate population has $N$ abundances that are too high with respect to the extreme groups. Thus, the likely explanation is that these are the warmest stars, responsible for the "flaring" of the scatter in $N$ seen above. This is simply an effect of the analysis and not a true feature in this cluster.

\section{Discussion and conclusions}

The present study highlights the advantage of having data for a large set of elements involved in the network of proton-capture reactions of H-burning at high temperature. It is very useful to have a simultaneous look at all relations among light elements because the depletions observed for $\mathrm{O}$ and $\mathrm{Mg}$, as well as the enhancements detected in $\mathrm{N}, \mathrm{Na}$, and $\mathrm{Al}$ allow the stratigraphy within the H-burning region to be probed, reaching more and more hot layers. In turn, this translates into evidence of more massive stars being responsible for intra-cluster pollution.

This large harvest of data can then be exploited to understand whether the formation of globular clusters was characterized by a single second episode or multiple episodes of star formation. Of course, when the observational evidence points toward only two distinct groups, as found in M 4 in the present and previous analyses (Ivans et al. 1999; Marino et al. 2008; Carretta et al. 2009a), the question must necessarily remain unanswered and the scenario is undetermined.

More interesting is the case when more than two groups are found. Leaving aside the peculiar case of $\omega$ Cen, the most noticeable case among normal GCs is NGC 2808. We know that this cluster a) shows three distinct main sequences (D'Antona et al. 2005; Piotto et al. 2007); b) has an unusual HB, bimodal and with a long blue tail (Walker 1999), that has been connected to different He enrichments (D'Antona \& Caloi 2004); and c) exhibits a very extended $\mathrm{Na}-\mathrm{O}$ anti-correlation, with some evidence of multiple "blobs" (Carretta et al. 2006).

Another more recent case is NGC 6752, where with our survey of $\mathrm{Al}$ abundances we found evidence of three distinct stellar populations with different chemical composition. Moreover, from the correlation between depleted species such as $\mathrm{O}$ and $\mathrm{Mg}$ (clear but not linear), we were able to show that the composition of the intermediate population cannot be obtained by simply mixing primordial matter with material having the chemical composition of the extreme population. 
The same approach can be followed to try to better understand our present finding in 47 Tuc. Using a simple dilution model (see Carretta et al. 2009a; Gratton et al. 2010), we searched for the dilution factors able to reproduce the intermediate group by mixing the typical composition of stars from the other two groups. If the model is correct, we should obtain the same factor, regardless of our choice of the element to be used. We derived that dilution factors $0.71,0.64$, and 0.63 (basically the same factor, within the uncertainties) are required for $\mathrm{Al}, \mathrm{O}$, and $\mathrm{Na}$, respectively. The $\mathrm{Mg}$ abundance variations are so small that virtually any factor applies. Our nominal $N$ abundances, which were obtained assuming a constant value of $[\mathrm{C} / \mathrm{Fe}]=-0.6$ for all stars, require a dilution factor of 0.29 that is quite different from the values obtained for the other elements. However, this result depends on the adoption of a constant $[\mathrm{C} / \mathrm{Fe}]$ and a different conclusion is obtained by assuming that the $\mathrm{C}$ abundance is different in the three groups. This is not at all an ad hoc assumption, since several studies have shown that the $[\mathrm{C} / \mathrm{Fe}]$ ratio is higher by $\sim 0.3$ dex in $\mathrm{CN}$-weak (Na-poor, O-rich) stars than in CN-strong (Na-rich, O-rich) stars (see Norris \& Freeman 1982; Norris \& Cottrell 1979; Smith et al. 1989; Brown et al. 1990; Briley 1997; Carretta et al. 2005). Were the $\mathrm{C}$ abundance in group 2 also intermediate between those of groups 1 and 2, it would be possible to reproduce the strength of the $\mathrm{CN}$ lines with a $N$ abundance compatible with a pollution scenario. Direct determination of $\mathrm{C}$ abundances for the stars in our sample would solve this ambiguity and clarify if dilution may explain the pattern seen in 47 Tuc. The same observation could also be used to find if the sum of $\mathrm{C}+\mathrm{N}+\mathrm{O}$ abundances is constant or not in 47 Tuc. We only note here that if we combine the $\mathrm{C}$ abundances for $\mathrm{CN}$-weak and $\mathrm{CN}$-strong RGB stars by Brown et al. (1990) and Briley (1997) ([C/Fe] -0.1 and $[\mathrm{C} / \mathrm{Fe}] \sim-0.3$, respectively) with our $\mathrm{CN}$ and $\mathrm{O}$ abundances, the sum of $\mathrm{C}+\mathrm{N}+\mathrm{O}$ abundances would be the same for the two extreme groups within $0.1 \mathrm{dex}$. This result agrees quite well with the study of the $\mathrm{C}+\mathrm{N}+\mathrm{O}$ sum in unevolved cluster stars in NGC 6752, NGC 6397, and 47 Tuc made by Carretta et al. (2005).

Another problem in 47 Tuc is to find the progeny of the SGB stars $\mathrm{C}+\mathrm{N}+\mathrm{O}$ enhanced, the so-called "SGII" hypothesised by Di Criscienzo et al. (2010), which are $10 \%$ of the stars. More investigations are needed to have a self-consistent picture of this cluster.

A promising approach could be to look for the full set of abundances, from C, N, O to the light and heavy, neutron-capture elements, especially if measured in the same stars. However, this is generally not available at the moment, so no stringent conclusions can be drawn. For instance, Worley et al. (2010) present a list of studies regarding heavy elements in 47 Tuc. Only in a few of them, and only for a limited number of stars, are abundances for the whole set of elements available (e.g, 12 TO and SGB stars in James et al. 2004, when combined with Carretta et al. 2005; four bright giants in Brown \& Wallerstein 1992). An interesting approach based on medium-resolution spectra of a large number of targets is presented in Worley \& Cottrel (2012), who obtained a measure of the $\mathrm{CN}$ excess for about 100 giants, with almost no overlap with our present sample. However, they did not measure $\mathrm{O}$ abundances and published results of the abundance analysis of other elements only for a small subsample of 13 bright stars. In all the above-mentioned studies, there seems to be a homogeneous pattern among elements produced by neutron-capture processes, and this conclusion seems to be a severe drawback for the hypothesis of a significant contribution of polluted matter by low-mass AGB stars in this cluster. We stress, however, that all the existing samples are too small to permit a separation of groups as we do in the present paper using the cluster-analysis technique. Observational efforts should be devoted to acquiring the most complete set of key-element abundances in significant samples of stars.

Our results show that 47 Tuc is the third normal GC after NGC 2808 (D’Antona et al. 2005; Piotto et al. 2007; Carretta et al. 2006) and NGC 6752 (Yong et al. 2008; Carretta et al. 2012a), where at least three groups of stars with homogeneous chemical composition (within each group) can be separated. The implication seems to be that for a fraction of GCs the scenario of cluster formation must include multiple bursts where second-generation stars are produced. In each, the combination of matter processed by polluters of different (obviously decreasing with time) average masses and pristine material gives the observed chemical pattern in each group. By studying the detailed abundance distribution of proton-capture elements, we can provide important constraints to the theoretical models of cluster formation.

All these ingredients must be carefully considered and incorporated into existing and future theoretical models. Homogeneous analysis of several proton-capture elements in large samples of stars such as the one presented in this study may provide a fruitful way to contribute useful constraints to these models.

Acknowledgements. This work was funded by the PRIN INAF 2009 grant CRA 1.06.12.10 "Formation and early evolution of massive star clusters", PI. R Gratton) and by the PRIN INAF 2011 grant "Multiple populations in globular clusters: their role in the Galaxy assembly" (PI Carretta). We thank Šarūnas Mikolaitis for sharing with us the line list for CN provided by B. Plez. This research made use of the SIMBAD database (in particular Vizier), operated at CDS, Strasbourg, France and of NASA's Astrophysical Data System.

\section{References}

Arnould, M., Goriely, S., \& Jorissen, A. 1999, A\&A, 347, 572

Bragaglia, A., Carretta, E., Gratton, R. G., et al. 2010, ApJ, 720, L41

Briley, M. M. 1997, AJ, 114, 1051

Brown, J. A., \& Wallerstein, G. 1992, AJ, 104, 1818

Brown, J. A., Wallerstein, G., \& Oke, J. B. 1990, AJ, 100, 1561

Carlberg, J. K., Cunha, K., Smith, V. V., \& Majewski, S. R. 2012, ApJ, 757, 109

Carretta, E., Gratton R. G., Bragaglia, A., Bonifacio, P., \& Pasquini, L. 2004, A\&A, 416, 925

Carretta, E., Gratton, R. G., Lucatello, S., Bragaglia, A., \& Bonifacio, P. 2005, A\&A, 433, 597

Carretta, E., Bragaglia, A., Gratton R. G., et al. 2006, A\&A, 450, 523

Carretta, E., Bragaglia, A., Gratton, R. G., et al. 2009a, A\&A, 505, 117

Carretta, E., Bragaglia, A., Gratton, R. G., \& Lucatello, S. 2009b, A\&A, 505, 139 (Paper VIII)

Carretta, E., Bragaglia, A., Gratton, R. G., D’Orazi, V., \& Lucatello, S. 2009c, A\&A, 508, 695

Carretta, E., Bragaglia, A., Gratton, R. G., et al. 2010, A\&A, 516, 55

Carretta, E., Lucatello, S., Gratton, R. G., Bragaglia, A., \& D’Orazi, V. 2011, A\&A, 533, 69

Carretta, E., Bragaglia, A., Gratton, R. G., Lucatello, S., \& D’Orazi, V. 2012a, ApJ, 750, L14

Carretta, E., D’Orazi, V., Gratton, R. G., \& Lucatello, S. 2012b, A\&A, 543, A117 D'Antona, F., \& Caloi, V. 2004, ApJ, 611, 871

D’Antona, F., Bellazzini, M., Caloi, V., et al. 2005, ApJ, 631, 868

D'Ercole, A., D'Antona, F., \& Vesperini, E. 2011, MNRAS, 415, 1304

Decressin, T., Meynet, G., Charbonnel C. Prantzos, N., \& Ekstrom, S. 2007, A\&A, 464, 1029

de Mink, S. E., Pols, O. R., Langer, N., \& Izzard, R. G. 2009, A\&A, 507, L1

Denisenkov, P. A., \& Denisenkova, S. N. 1989, A. Tsir., 1538, 11

Di Criscienzo, M., Ventura, P., D’Antona, F., Milone, A., \& Piotto, G. 2010, MNRAS, 408, 999

D’Orazi, V., Gratton, R. G., Pancino, E., et al. 2011, A\&A, 534, A29

Gratton, R. G. 1988, Rome Obs. Preprint Ser., 29

Gratton, R. G., Bonifacio, P., Bragaglia, A., et al. 2001, A\&A, 369, 87

Gratton, R. G., Sneden, C., \& Carretta, E. 2004, ARA\&A, 42, 385 
Gratton, R. G., Carretta, E., Bragaglia, A., Lucatello, S., \& D’Orazi, V. 2010, A\&A, 517, 81

Gratton, R. G., Carretta, E., \& Bragaglia, A. 2012, A\&ARv, 20, 50

Gratton, R. G., Lucatello, S., Sollima, A., et al. 2013, A\&A, 549, A41

Harris, W. 1996, AJ, 112, 1487

Hinkle, K., Wallace, L., Valenti, J., \& Harmer, D. 2000, Visible and Near Infrared Atlas of the Arcturus Spectrum 3727-9300 A (San Francisco: ASP)

Ivans, I. I., Sneden, C., Kraft, R. P., et al. 1999, AJ, 118, 1273

James, G., François, P., Bonifacio, P., et al. 2004, A\&A, 427, 825

Kraft, R. P. 1994, PASP, 106, 553

Kurucz, R. L. 1993, CD-ROM 13, Smithsonian Astrophysical Observatory, Cambridge

Langer, G. E., Hoffman, R., \& Sneden, C. 1993, PASP, 105, 301

MacQueen, J. B. 1967, in Mathematical Statistics and Probability (University of California Press), 281

Marino, A. F., Villanova, S., Piotto, G., et al. 2008, A\&A, 490, 625

Martell, S. L. 2011, Astron. Nachr., 332, 467

Milone, A., Piotto, G., Bedin, L., et al. 2012, ApJ, 744, 58

Norris, J. E., \& Cottrell, P. L. 1979, ApJ, 229, 69
Norris, J., \& Freeman, K. C. 1979, ApJ, 230, L179

Norris, J. E., \& Freeman, K. C. 1982, ApJ, 254, 143

Piotto, G., Bedin, L. R., Anderson, J., et al. 2007, ApJ, 661, L53

Prantzos, N., \& Charbonnel, C. 2006, A\&A, 458, 135

$\mathrm{R}$ Development Core Team 2011, R: A language and environment for statistical computing (Vienna, Austria: R Foundation for Statistical Computing)

Ramirez, S., \& Cohen, J. G. 2002, AJ, 123, 3277

Smith, G. H., \& Briley, M. M. 2005, PASP, 117, 895

Smith, G. H., Bell, R. A., \& Hesser, J. E. 1989, ApJ, 341, 190

Steinhaus, H. 1956, Bull. Acad. Polon. Sci. 4, 801

Ventura, P., D’Antona, F., Mazzitelli, I., \& Gratton, R. 2001, ApJ, 550, L65

Walker, A. R. 1999, AJ, 118, 432

Worley, C. C., \& Cottrell, P. L. 2012, PASA, 29, 29

Worley, C. C., Cottrell, P. L., McDonald, I., \& van Loon, J. T. 2010, MNRAS, 402, 2060

Yong, D., Grundahl, F., Nissen, P. E., Jensen, H. R., \& Lambert, D. L. 2005 , A\&A, 438, 875

Yong, D., Grundahl, F., Johnson, J. A., \& Asplund, M. 2008, ApJ, 684, 1159 
E. Carretta et al.: Aluminium abundances in 47 Tuc and M 4

Table 2. Derived abundances in 47 Tuc.

\begin{tabular}{|c|c|c|c|c|c|c|c|c|c|c|c|c|}
\hline Star & $\mathrm{nr}^{a}$ & $\begin{array}{c}{[\mathrm{N} / \mathrm{Fe}]} \\
(\mathrm{dex})\end{array}$ & $\begin{array}{l}\text { rms } \\
(\mathrm{dex})\end{array}$ & $\begin{array}{c}{[\mathrm{Al} / \mathrm{Fe}]} \\
(\mathrm{dex})\end{array}$ & $\begin{array}{l}\mathrm{err}^{b} \\
(\mathrm{dex})\end{array}$ & $\mathrm{S} / \mathrm{N}$ & $n^{c}$ & {$[\mathrm{Mg} / \mathrm{Fe}]$} & $\mathrm{rms}$ & $n^{c}$ & {$[\mathrm{Si} / \mathrm{Fe}]$} & rms \\
\hline 1389 & & & & & & & 2 & 0.64 & 0.05 & 2 & 0.32 & 0.05 \\
\hline 2608 & 18 & 1.03 & 0.20 & 0.48 & 0.01 & 346 & 3 & 0.63 & 0.09 & 7 & 0.47 & 0.14 \\
\hline 2871 & 17 & 1.15 & 0.15 & 0.67 & 0.02 & 150 & 1 & 0.46 & & 10 & 0.41 & 0.17 \\
\hline 4373 & 17 & 0.83 & 0.08 & 0.31 & 0.01 & 214 & 3 & 0.60 & 0.05 & 12 & 0.48 & 0.20 \\
\hline 5172 & 18 & 1.11 & 0.13 & 0.46 & 0.02 & 187 & 3 & 0.47 & 0.07 & 12 & 0.49 & 0.12 \\
\hline 5270 & & & & & & & 2 & 0.69 & 0.08 & 2 & 0.43 & 0.20 \\
\hline 5277 & 18 & 0.82 & 0.10 & 0.41 & 0.01 & 265 & 3 & 0.49 & 0.25 & 7 & 0.40 & 0.10 \\
\hline 5640 & 16 & 0.93 & 0.06 & 0.45 & 0.02 & 170 & 3 & 0.40 & 0.10 & 11 & 0.43 & 0.20 \\
\hline 6092 & 17 & 0.85 & 0.09 & 0.43 & 0.01 & 358 & 1 & 0.58 & & 8 & 0.40 & 0.15 \\
\hline 6808 & 18 & 1.02 & 0.12 & 0.53 & 0.00 & 998 & 3 & 0.47 & 0.09 & 11 & 0.41 & 0.11 \\
\hline 7711 & 17 & 1.02 & 0.13 & 0.58 & 0.03 & 104 & 3 & 0.44 & 0.15 & 11 & 0.40 & 0.21 \\
\hline 7904 & 17 & 1.14 & 0.10 & 0.56 & 0.02 & 192 & 3 & 0.42 & 0.01 & 10 & 0.33 & 0.09 \\
\hline 9163 & 17 & 0.96 & 0.11 & 0.59 & 0.02 & 173 & 1 & 0.64 & & 8 & 0.41 & 0.22 \\
\hline 9268 & & & & & & & 2 & 0.52 & 0.08 & 2 & 0.52 & 0.03 \\
\hline 9518 & 18 & 0.87 & 0.08 & 0.38 & 0.02 & 136 & 3 & 0.52 & 0.14 & 5 & 0.32 & 0.09 \\
\hline 9717 & 18 & 0.91 & 0.10 & 0.45 & 0.01 & 278 & 3 & 0.48 & 0.14 & 11 & 0.36 & 0.13 \\
\hline 10397 & 17 & 0.98 & 0.09 & 0.48 & 0.02 & 124 & 3 & 0.48 & 0.16 & 8 & 0.36 & 0.11 \\
\hline 10496 & 17 & 1.14 & 0.17 & 0.56 & 0.01 & 241 & 3 & 0.42 & 0.08 & 11 & 0.39 & 0.15 \\
\hline 10994 & 15 & 0.81 & 0.08 & 0.23 & 0.02 & 221 & 3 & 0.43 & 0.13 & 12 & 0.49 & 0.13 \\
\hline 11008 & 17 & 1.06 & 0.12 & 0.51 & 0.02 & 171 & 3 & 0.40 & 0.05 & 12 & 0.39 & 0.15 \\
\hline 11169 & 18 & 1.00 & 0.12 & 0.45 & 0.01 & 244 & 3 & 0.60 & 0.05 & 9 & 0.37 & 0.11 \\
\hline 11675 & 18 & 1.20 & 0.20 & 0.47 & 0.02 & 128 & 3 & 0.67 & 0.15 & 12 & 0.46 & 0.14 \\
\hline 11709 & 18 & 1.20 & 0.16 & 0.72 & 0.01 & 295 & 3 & 0.64 & 0.12 & 7 & 0.37 & 0.11 \\
\hline 11756 & 18 & 0.92 & 0.08 & 0.46 & 0.02 & 147 & 3 & 0.57 & 0.18 & 11 & 0.44 & 0.13 \\
\hline 11927 & 18 & 1.11 & 0.12 & 0.55 & 0.00 & 677 & 3 & 0.46 & 0.12 & 10 & 0.44 & 0.09 \\
\hline 12128 & 18 & 0.94 & 0.10 & 0.36 & 0.00 & 1665 & 1 & 0.59 & & 7 & 0.38 & 0.14 \\
\hline 12186 & 17 & 0.81 & 0.14 & 0.42 & 0.01 & 223 & 1 & 0.54 & & 7 & 0.42 & 0.18 \\
\hline 12272 & 17 & 0.98 & 0.12 & 0.40 & 0.01 & 610 & 1 & 0.56 & & 8 & 0.56 & 0.16 \\
\hline 13047 & 17 & 0.81 & 0.09 & 0.40 & 0.00 & 992 & 3 & 0.52 & 0.14 & 10 & 0.41 & 0.15 \\
\hline 13668 & 17 & 0.86 & 0.12 & 0.40 & 0.01 & 290 & 3 & 0.48 & 0.08 & 10 & 0.37 & 0.08 \\
\hline 13718 & 17 & 0.80 & 0.11 & 0.51 & 0.01 & 288 & 3 & 0.54 & 0.18 & 11 & 0.48 & 0.21 \\
\hline 13795 & & & & & & & 2 & 0.47 & 0.04 & 2 & 0.56 & 0.00 \\
\hline 13939 & & & & & & & 2 & 0.63 & 0.06 & 2 & 0.37 & 0.08 \\
\hline 14262 & 18 & 0.90 & 0.12 & 0.48 & 0.02 & 176 & 3 & 0.59 & 0.12 & 10 & 0.45 & 0.16 \\
\hline 14487 & 17 & 0.92 & 0.10 & 0.42 & 0.01 & 197 & 3 & 0.60 & 0.11 & 11 & 0.43 & 0.12 \\
\hline 14496 & & & & & & & 2 & 0.47 & 0.02 & 1 & 0.37 & \\
\hline 14583 & & & & & & & 2 & 0.47 & 0.14 & 2 & 0.44 & 0.09 \\
\hline 15451 & & & & & & & 2 & 0.53 & 0.06 & 2 & 0.45 & 0.09 \\
\hline 15552 & 18 & 1.22 & 0.20 & 0.62 & 0.02 & 182 & 3 & 0.66 & 0.07 & 7 & 0.39 & 0.12 \\
\hline 15695 & 18 & 1.09 & 0.18 & 0.47 & 0.01 & 267 & 1 & 0.42 & & 10 & 0.44 & 0.10 \\
\hline 16089 & & & & & & & 2 & 0.68 & 0.07 & 2 & 0.35 & 0.09 \\
\hline 16462 & 18 & 1.10 & 0.10 & 0.74 & 0.01 & 390 & 3 & 0.47 & 0.12 & 10 & 0.37 & 0.16 \\
\hline 16597 & 18 & 1.14 & 0.12 & 0.43 & 0.03 & 95 & 3 & 0.68 & 0.06 & 10 & 0.34 & 0.18 \\
\hline 16777 & 18 & 1.05 & 0.13 & 0.63 & 0.01 & 390 & 3 & 0.47 & 0.10 & 12 & 0.44 & 0.14 \\
\hline 16829 & 18 & 1.14 & 0.10 & 0.52 & 0.03 & 265 & 3 & 0.61 & 0.03 & 9 & 0.48 & 0.17 \\
\hline 16957 & & & & & & & 2 & 0.48 & 0.09 & 2 & 0.49 & 0.06 \\
\hline 17193 & 18 & 0.86 & 0.10 & 0.48 & 0.01 & 218 & 3 & 0.58 & 0.13 & 7 & 0.51 & 0.13 \\
\hline 17238 & 17 & 1.11 & 0.19 & 0.69 & 0.02 & 208 & 3 & 0.65 & 0.11 & 12 & 0.44 & 0.14 \\
\hline 17526 & 17 & 1.03 & 0.15 & 0.66 & 0.02 & 177 & 3 & 0.56 & 0.09 & 10 & 0.41 & 0.13 \\
\hline 17657 & 18 & 1.17 & 0.20 & 0.80 & 0.01 & 501 & 1 & 0.55 & & 7 & 0.36 & 0.24 \\
\hline 17885 & 18 & 1.15 & 0.13 & 0.57 & 0.01 & 394 & 1 & 0.58 & & 8 & 0.43 & 0.22 \\
\hline 17926 & 17 & 1.14 & 0.15 & 0.63 & 0.01 & 626 & 3 & 0.52 & 0.10 & 12 & 0.57 & 0.16 \\
\hline 18374 & 17 & 1.19 & 0.14 & 0.68 & 0.03 & 93 & 3 & 0.41 & 0.10 & 9 & 0.34 & 0.11 \\
\hline 18623 & 17 & 1.03 & 0.12 & 0.58 & 0.01 & 547 & 1 & 0.68 & & 7 & 0.39 & 0.16 \\
\hline 18676 & 17 & 1.12 & 0.11 & 0.41 & 0.01 & 282 & 1 & 0.51 & & 9 & 0.35 & 0.21 \\
\hline 18896 & 15 & 1.21 & 0.13 & 0.67 & 0.03 & 114 & 3 & 0.63 & 0.02 & 11 & 0.48 & 0.15 \\
\hline 19042 & 18 & 1.08 & 0.17 & 0.93 & 0.00 & 946 & 3 & 0.55 & 0.06 & 9 & 0.44 & 0.24 \\
\hline 19394 & 16 & 0.81 & 0.12 & 0.41 & 0.02 & 167 & 1 & 0.56 & & 10 & 0.48 & 0.16 \\
\hline 19992 & 18 & 0.81 & 0.08 & 0.38 & 0.01 & 212 & 3 & 0.45 & 0.18 & 12 & 0.35 & 0.13 \\
\hline 20002 & 18 & 1.05 & 0.12 & 0.52 & 0.01 & 492 & 1 & 0.66 & & 7 & 0.44 & 0.23 \\
\hline 20019 & 16 & 0.98 & 0.06 & 0.53 & 0.01 & 204 & 3 & 0.63 & 0.12 & 11 & 0.45 & 0.14 \\
\hline 20387 & 17 & 1.05 & 0.10 & 0.53 & 0.01 & 280 & 3 & 0.52 & 0.13 & 12 & 0.49 & 0.11 \\
\hline 20639 & 14 & 1.15 & 0.08 & 0.56 & 0.02 & 170 & 1 & 0.51 & & 10 & 0.45 & 0.18 \\
\hline
\end{tabular}

Notes. ${ }^{(a)}$ Number of $\mathrm{CN}$ features used to derive $[\mathrm{N} / \mathrm{Fe}] .{ }^{(b)}$ Errors in the abundance of Al due to errors in flux. ${ }^{(c)}$ Number of lines. 
Table 2. continued.

\begin{tabular}{|c|c|c|c|c|c|c|c|c|c|c|c|c|}
\hline Star & $\mathrm{nr}^{a}$ & {$[\mathrm{~N} / \mathrm{Fe}]$} & $\mathrm{rms}$ & {$[\mathrm{Al} / \mathrm{Fe}]$} & $\mathrm{err}^{b}$ & $\mathrm{~S} / \mathrm{N}$ & $n^{c}$ & {$[\mathrm{Mg} / \mathrm{Fe}]$} & $\mathrm{rms}$ & $n^{c}$ & {$[\mathrm{Si} / \mathrm{Fe}]$} & $\mathrm{rms}$ \\
\hline 20701 & 18 & 1.17 & 0.19 & 0.59 & 0.07 & 352 & 3 & 0.46 & 0.07 & 12 & 0.44 & 0.12 \\
\hline 20983 & & & & & & & 2 & 0.43 & 0.07 & 2 & 0.46 & 0.06 \\
\hline 21369 & 18 & 1.25 & 0.21 & 0.69 & 0.02 & 188 & 3 & 0.62 & 0.19 & 12 & 0.49 & 0.11 \\
\hline 22374 & & & & & & & 2 & 0.43 & 0.04 & 2 & 0.42 & 0.10 \\
\hline 22726 & 17 & 1.02 & 0.12 & 0.61 & 0.01 & 525 & 3 & 0.46 & 0.07 & 12 & 0.50 & 0.18 \\
\hline 22875 & 18 & 1.06 & 0.18 & 0.47 & 0.03 & 99 & 1 & 0.51 & & 10 & 0.38 & 0.23 \\
\hline 23175 & 18 & 1.10 & 0.16 & 0.60 & 0.00 & 1233 & 3 & 0.62 & 0.27 & 8 & 0.43 & 0.10 \\
\hline 23211 & 18 & 1.16 & 0.13 & 0.77 & 0.02 & 145 & 3 & 0.60 & 0.11 & 12 & 0.51 & 0.16 \\
\hline 23225 & 16 & 0.89 & 0.12 & 0.32 & 0.02 & 153 & 1 & 0.54 & & 9 & 0.39 & 0.21 \\
\hline 23232 & 17 & 0.90 & 0.11 & 0.40 & 0.02 & 189 & 2 & 0.53 & 0.02 & 2 & 0.24 & 0.00 \\
\hline 23236 & 18 & 1.00 & 0.22 & 0.59 & 0.01 & 262 & 3 & 0.60 & 0.06 & 12 & 0.44 & 0.20 \\
\hline 23335 & 18 & 1.05 & 0.14 & 0.57 & 0.01 & 360 & 3 & 0.47 & 0.09 & 7 & 0.40 & 0.13 \\
\hline 23782 & 15 & 1.08 & 0.11 & 0.57 & 0.02 & 175 & 1 & 0.60 & & 5 & 0.51 & 0.19 \\
\hline 23799 & 18 & 1.12 & 0.17 & 0.33 & 0.11 & 27 & 1 & 0.40 & & 7 & 0.38 & 0.07 \\
\hline 23821 & & & & & & & 2 & 0.43 & 0.13 & 2 & 0.51 & 0.14 \\
\hline 23829 & 17 & 0.87 & 0.06 & 0.41 & 0.01 & 320 & 1 & 0.54 & & 10 & 0.56 & 0.17 \\
\hline 23879 & & & & & & & 3 & 0.57 & 0.21 & 7 & 0.57 & 0.16 \\
\hline 24463 & 17 & 1.14 & 0.20 & 0.74 & 0.01 & 263 & 3 & 0.65 & 0.04 & 11 & 0.50 & 0.21 \\
\hline 24533 & 16 & 1.11 & 0.17 & 0.62 & 0.01 & 206 & 3 & 0.64 & 0.09 & 13 & 0.37 & 0.28 \\
\hline 24553 & & & & & & & 2 & 0.67 & 0.09 & 2 & 0.33 & 0.01 \\
\hline 24665 & & & & & & & 2 & 0.60 & 0.03 & 2 & 0.40 & 0.09 \\
\hline 24901 & 18 & 1.14 & 0.14 & 1.04 & 0.02 & 210 & 1 & 0.57 & & 10 & 0.56 & 0.16 \\
\hline 25226 & & & & & & & 2 & 0.53 & 0.08 & 2 & 0.57 & 0.14 \\
\hline 25487 & 16 & 1.11 & 0.12 & 0.50 & 0.01 & 228 & 3 & 0.59 & 0.05 & 12 & 0.45 & 0.18 \\
\hline 25770 & 16 & 1.20 & 0.15 & 0.62 & 0.01 & 550 & 3 & 0.53 & 0.09 & 12 & 0.48 & 0.11 \\
\hline 25961 & & & & & & & 3 & 0.66 & 0.06 & 9 & 0.42 & 0.13 \\
\hline 25988 & 15 & 1.14 & 0.06 & 0.63 & 0.01 & 275 & 1 & 0.54 & & 6 & 0.37 & 0.07 \\
\hline 26058 & 18 & 0.97 & 0.12 & 0.33 & 0.01 & 312 & 3 & 0.59 & 0.01 & 6 & 0.40 & 0.15 \\
\hline 26281 & 17 & 1.17 & 0.16 & 0.58 & 0.02 & 161 & 3 & 0.43 & 0.08 & 11 & 0.40 & 0.10 \\
\hline 26403 & & & & & & & 2 & 0.47 & 0.09 & 2 & 0.53 & 0.02 \\
\hline 26713 & 18 & 1.06 & 0.20 & 0.47 & 0.01 & 497 & 3 & 0.44 & 0.09 & 12 & 0.39 & 0.08 \\
\hline 26870 & 17 & 1.14 & 0.10 & 0.65 & 0.01 & 267 & 3 & 0.59 & 0.08 & 9 & 0.46 & 0.14 \\
\hline 27381 & 18 & 1.17 & 0.15 & 0.71 & 0.01 & 250 & 3 & 0.68 & 0.03 & 11 & 0.56 & 0.32 \\
\hline 27385 & 18 & 1.00 & 0.15 & 0.53 & 0.00 & 846 & 3 & 0.53 & 0.28 & 7 & 0.47 & 0.20 \\
\hline 27499 & 17 & 1.12 & 0.11 & 0.51 & 0.02 & 149 & 3 & 0.43 & 0.01 & 11 & 0.43 & 0.08 \\
\hline 27551 & & & & & & & 2 & 0.57 & 0.07 & 2 & 0.37 & 0.03 \\
\hline 27755 & 17 & 1.02 & 0.12 & 0.45 & 0.02 & 122 & 3 & 0.56 & 0.06 & 8 & 0.40 & 0.19 \\
\hline 28628 & 18 & 1.11 & 0.11 & 0.42 & 0.01 & 390 & 1 & 0.47 & & 10 & 0.48 & 0.11 \\
\hline 29031 & 18 & 0.90 & 0.15 & 0.46 & 0.02 & 193 & 1 & 0.33 & & 7 & 0.38 & 0.10 \\
\hline 29167 & 16 & 1.09 & 0.10 & 0.59 & 0.01 & 427 & 1 & 0.60 & & 9 & 0.52 & 0.19 \\
\hline 29490 & 17 & 0.81 & 0.09 & 0.37 & 0.02 & 190 & 3 & 0.51 & 0.08 & 12 & 0.39 & 0.15 \\
\hline 29886 & & & & & & & 2 & 0.45 & 0.12 & 2 & 0.39 & 0.03 \\
\hline 30104 & 17 & 1.15 & 0.11 & 0.65 & 0.01 & 400 & 3 & 0.44 & 0.06 & 12 & 0.52 & 0.11 \\
\hline 30193 & 18 & 0.97 & 0.10 & 0.52 & 0.01 & 339 & 1 & 0.51 & & 10 & 0.43 & 0.10 \\
\hline 30297 & 17 & 0.85 & 0.09 & 0.42 & 0.01 & 406 & 3 & 0.41 & 0.09 & 12 & 0.42 & 0.10 \\
\hline 30340 & & & & & & & 2 & 0.61 & 0.04 & 2 & 0.48 & 0.22 \\
\hline 30463 & 18 & 0.99 & 0.12 & 0.64 & 0.01 & 224 & 3 & 0.49 & 0.19 & 11 & 0.52 & 0.25 \\
\hline 30949 & 16 & 1.10 & 0.20 & 0.62 & 0.03 & 97 & 3 & 0.44 & 0.08 & 12 & 0.37 & 0.09 \\
\hline 30952 & & & & & & & 2 & 0.45 & 0.06 & 2 & 0.40 & 0.02 \\
\hline 31100 & 17 & 1.08 & 0.13 & 0.42 & 0.02 & 189 & 1 & 0.64 & & 10 & 0.43 & 0.17 \\
\hline 31190 & 18 & 1.16 & 0.11 & 0.42 & 0.01 & 512 & 3 & 0.65 & 0.08 & 7 & 0.41 & 0.17 \\
\hline 31223 & 18 & 1.06 & 0.17 & 0.54 & 0.02 & 161 & 1 & 0.58 & & 10 & 0.49 & 0.30 \\
\hline 31426 & 17 & 1.08 & 0.14 & 0.52 & 0.00 & 694 & 3 & 0.49 & 0.16 & 10 & 0.45 & 0.20 \\
\hline 31525 & & & & & & & 2 & 0.53 & 0.06 & 2 & 0.50 & 0.09 \\
\hline 31560 & 17 & 0.94 & 0.08 & 0.41 & 0.01 & 349 & 3 & 0.52 & 0.07 & 10 & 0.41 & 0.15 \\
\hline 31638 & 17 & 1.08 & 0.11 & 0.53 & 0.02 & 191 & 1 & 0.46 & & 10 & 0.46 & 0.13 \\
\hline 31796 & 18 & 1.26 & 0.13 & 0.75 & 0.02 & 143 & 1 & 0.65 & & 10 & 0.49 & 0.16 \\
\hline 32730 & 18 & 1.11 & 0.13 & 0.67 & 0.01 & 563 & 3 & 0.47 & 0.11 & 7 & 0.51 & 0.09 \\
\hline 33009 & 18 & 1.11 & 0.13 & 0.36 & 0.01 & 249 & 3 & 0.46 & 0.05 & 10 & 0.45 & 0.07 \\
\hline 33311 & & & & 1.11 & 0.01 & 434 & 1 & 0.61 & & 5 & 0.52 & 0.19 \\
\hline 33686 & & & & & & & 2 & 0.54 & 0.06 & 2 & 0.35 & 0.03 \\
\hline 34033 & 18 & 1.06 & 0.10 & 0.50 & 0.01 & 452 & 3 & 0.49 & 0.14 & 10 & 0.41 & 0.13 \\
\hline 34847 & 18 & 0.88 & 0.08 & 0.44 & 0.00 & 727 & 1 & 0.55 & & 7 & 0.58 & 0.17 \\
\hline 35160 & 18 & 1.19 & 0.15 & 0.75 & 0.01 & 601 & 3 & 0.50 & 0.13 & 11 & 0.51 & 0.22 \\
\hline
\end{tabular}


E. Carretta et al.: Aluminium abundances in 47 Tuc and M 4

Table 2. continued.

\begin{tabular}{|c|c|c|c|c|c|c|c|c|c|c|c|c|}
\hline Star & $\mathrm{nr}^{a}$ & {$[\mathrm{~N} / \mathrm{Fe}]$} & $\mathrm{rms}$ & {$[\mathrm{Al} / \mathrm{Fe}]$} & $\mathrm{err}^{b}$ & $\mathrm{~S} / \mathrm{N}$ & $n^{c}$ & {$[\mathrm{Mg} / \mathrm{Fe}]$} & $\mathrm{rms}$ & $n^{c}$ & {$[\mathrm{Si} / \mathrm{Fe}]$} & $\mathrm{rms}$ \\
\hline 35454 & & & & & & & 2 & 0.51 & 0.01 & 2 & 0.53 & 0.01 \\
\hline 35878 & 17 & 1.07 & 0.15 & 0.63 & 0.01 & 310 & 3 & 0.47 & 0.17 & 10 & 0.44 & 0.10 \\
\hline 36828 & & & & & & & 2 & 0.45 & 0.02 & 2 & 0.55 & 0.13 \\
\hline 37009 & 16 & 1.15 & 0.18 & 0.52 & 0.01 & 221 & 1 & 0.48 & & 10 & 0.47 & 0.10 \\
\hline 37036 & 17 & 0.98 & 0.12 & 0.40 & 0.01 & 260 & 3 & 0.41 & 0.09 & 12 & 0.45 & 0.11 \\
\hline 37233 & 17 & 1.15 & 0.11 & 0.71 & 0.02 & 183 & 3 & 0.53 & 0.09 & 11 & 0.45 & 0.21 \\
\hline 38289 & 17 & 0.85 & 0.09 & 0.38 & 0.01 & 247 & 3 & 0.42 & 0.03 & 11 & 0.36 & 0.12 \\
\hline 38976 & 18 & 0.96 & 0.08 & 0.39 & 0.01 & 357 & 1 & 0.60 & & 8 & 0.38 & 0.18 \\
\hline 39101 & 18 & 1.07 & 0.16 & 0.58 & 0.01 & 678 & 3 & 0.48 & 0.20 & 8 & 0.44 & 0.16 \\
\hline 39836 & 18 & 0.84 & 0.11 & 0.35 & 0.03 & 84 & 1 & 0.60 & & 6 & 0.43 & 0.17 \\
\hline 40001 & 18 & 1.09 & 0.19 & 0.50 & 0.02 & 164 & 1 & 0.66 & & 10 & 0.39 & 0.22 \\
\hline 40718 & & & & & & & 2 & 0.51 & 0.09 & 1 & 0.44 & \\
\hline 41654 & 18 & 1.08 & 0.15 & 0.53 & 0.01 & 338 & 1 & 0.59 & & 7 & 0.53 & 0.12 \\
\hline 42243 & & & & & & & 2 & 0.56 & 0.09 & 2 & 0.52 & 0.02 \\
\hline 42321 & 18 & 0.94 & 0.17 & 0.31 & 0.02 & 147 & 1 & 0.56 & & 10 & 0.47 & 0.21 \\
\hline 42866 & 18 & 1.01 & 0.12 & 0.51 & 0.02 & 200 & 3 & 0.47 & 0.11 & 12 & 0.55 & 0.17 \\
\hline 43632 & 17 & 1.07 & 0.12 & 0.56 & 0.02 & 184 & 3 & 0.48 & 0.12 & 10 & 0.40 & 0.16 \\
\hline 43852 & 18 & 1.09 & 0.16 & 0.60 & 0.01 & 420 & 3 & 0.48 & 0.05 & 12 & 0.53 & 0.15 \\
\hline 43889 & 16 & 1.17 & 0.08 & 0.63 & 0.01 & 278 & 3 & 0.48 & 0.05 & 9 & 0.53 & 0.12 \\
\hline 112186 & & & & & & & 2 & 0.51 & 0.05 & 2 & 0.45 & 0.34 \\
\hline
\end{tabular}


Table 3. Derived abundances in M 4.

\begin{tabular}{|c|c|c|c|c|c|c|c|c|c|c|c|c|}
\hline Star & $\mathrm{nr}^{a}$ & $\begin{array}{c}{[\mathrm{N} / \mathrm{Fe}]} \\
(\mathrm{dex})\end{array}$ & $\begin{array}{l}\mathrm{rms} \\
(\mathrm{dex})\end{array}$ & $\begin{array}{c}{[\mathrm{Al} / \mathrm{Fe}]} \\
(\mathrm{dex})\end{array}$ & $\begin{array}{l}\operatorname{err}^{b} \\
(\operatorname{dex})\end{array}$ & $\mathrm{S} / \mathrm{N}$ & $n^{c}$ & {$[\mathrm{Mg} / \mathrm{Fe}]$} & rms & $n^{c}$ & {$[\mathrm{Si} / \mathrm{Fe}]$} & $\mathrm{rms}$ \\
\hline 5003 & 17 & 0.94 & 0.08 & 0.85 & 0.02 & 136 & 3 & 0.59 & 0.24 & 6 & 0.49 & 0.10 \\
\hline 5359 & 18 & 1.04 & 0.16 & 0.53 & 0.01 & 222 & 3 & 0.60 & 0.08 & 7 & 0.52 & 0.16 \\
\hline 8195 & & & & & & & 2 & 0.55 & 0.03 & 2 & 0.50 & 0.04 \\
\hline 20073 & 17 & 1.30 & 0.27 & 0.77 & 0.03 & 115 & 3 & 0.56 & 0.10 & 9 & 0.56 & 0.08 \\
\hline 20287 & 18 & 1.28 & 0.16 & 0.84 & 0.02 & 146 & 3 & 0.52 & 0.02 & 10 & 0.52 & 0.11 \\
\hline 20766 & 17 & 1.04 & 0.08 & 0.77 & 0.01 & 207 & 1 & 0.58 & & 7 & 0.51 & 0.12 \\
\hline 21493 & 17 & 1.15 & 0.24 & 0.94 & 0.02 & 161 & 1 & 0.50 & & 5 & 0.55 & 0.15 \\
\hline 21677 & 16 & 1.07 & 0.14 & 0.76 & 0.04 & 79 & 1 & 0.49 & & 8 & 0.54 & 0.19 \\
\hline 21728 & 18 & 1.04 & 0.08 & 0.77 & 0.02 & 145 & 1 & 0.47 & & 9 & 0.54 & 0.10 \\
\hline 22089 & 18 & 1.15 & 0.10 & 0.74 & 0.03 & 117 & 3 & 0.57 & 0.07 & 11 & 0.58 & 0.09 \\
\hline 22420 & 17 & 0.86 & 0.09 & 0.38 & 0.03 & 112 & 1 & 0.55 & & 8 & 0.46 & 0.08 \\
\hline 23052 & 18 & 1.04 & 0.21 & 0.81 & 0.03 & 107 & 3 & 0.53 & 0.15 & 7 & 0.54 & 0.14 \\
\hline 24374 & 18 & 1.26 & 0.23 & 0.74 & 0.02 & 147 & 2 & 0.44 & 0.05 & 9 & 0.51 & 0.14 \\
\hline 25709 & 18 & 1.03 & 0.13 & 0.73 & 0.01 & 222 & 3 & 0.50 & 0.15 & 10 & 0.59 & 0.06 \\
\hline 26471 & 18 & 0.90 & 0.13 & 0.59 & 0.01 & 232 & 1 & 0.56 & & 6 & 0.49 & 0.16 \\
\hline 26794 & 18 & 1.04 & 0.16 & 0.61 & 0.01 & 227 & 3 & 0.61 & 0.03 & 12 & 0.55 & 0.15 \\
\hline 27448 & 18 & 0.81 & 0.08 & 0.49 & 0.01 & 321 & 1 & 0.49 & & 7 & 0.48 & 0.07 \\
\hline 28356 & & & & & & & 2 & 0.50 & 0.07 & 2 & 0.58 & 0.00 \\
\hline 28707 & 18 & 1.11 & 0.16 & 0.69 & 0.02 & 129 & 3 & 0.61 & 0.02 & 8 & 0.55 & 0.16 \\
\hline 28797 & 18 & 1.12 & 0.13 & 0.81 & 0.01 & 210 & 3 & 0.52 & 0.05 & 9 & 0.57 & 0.08 \\
\hline 28847 & 18 & 0.95 & 0.14 & 0.55 & 0.01 & 215 & 3 & 0.48 & 0.02 & 12 & 0.51 & 0.23 \\
\hline 28977 & 18 & 1.07 & 0.15 & 0.71 & 0.02 & 187 & 1 & 0.44 & & 9 & 0.56 & 0.08 \\
\hline 29027 & 17 & 0.92 & 0.17 & 0.56 & 0.02 & 182 & 3 & 0.51 & 0.09 & 12 & 0.53 & 0.13 \\
\hline 29065 & & & & & & & 2 & 0.55 & 0.03 & 2 & 0.50 & 0.02 \\
\hline 29171 & 18 & 1.12 & 0.22 & 0.82 & 0.02 & 147 & 3 & 0.48 & 0.10 & 10 & 0.51 & 0.11 \\
\hline 29222 & 18 & 1.02 & 0.21 & 0.70 & 0.02 & 160 & 3 & 0.58 & 0.11 & 10 & 0.60 & 0.08 \\
\hline 29272 & 17 & 0.85 & 0.09 & 0.58 & 0.01 & 233 & 3 & 0.50 & 0.03 & 10 & 0.51 & 0.13 \\
\hline 29282 & 17 & 1.08 & 0.10 & 0.78 & 0.01 & 214 & 3 & 0.60 & 0.03 & 11 & 0.54 & 0.19 \\
\hline 29598 & 16 & 1.31 & 0.13 & 0.70 & 0.02 & 162 & 1 & 0.51 & & 6 & 0.56 & 0.18 \\
\hline 29693 & & & & & & & 2 & 0.45 & 0.02 & 2 & 0.62 & 0.00 \\
\hline 29848 & 18 & 0.94 & 0.13 & 0.85 & 0.01 & 214 & 1 & 0.63 & & 9 & 0.55 & 0.19 \\
\hline 30083 & 16 & 1.14 & 0.13 & 0.76 & 0.02 & 182 & 3 & 0.56 & 0.07 & 11 & 0.53 & 0.12 \\
\hline 30209 & & & & & & & 2 & 0.55 & 0.05 & 2 & 0.50 & 0.07 \\
\hline 30345 & 17 & 1.26 & 0.24 & 0.81 & 0.02 & 135 & 1 & 0.60 & & 6 & 0.52 & 0.10 \\
\hline 30381 & 17 & 1.03 & 0.17 & 0.81 & 0.02 & 165 & 3 & 0.59 & 0.19 & 8 & 0.57 & 0.15 \\
\hline 30450 & 17 & 1.09 & 0.09 & 0.87 & 0.02 & 173 & 2 & 0.57 & 0.03 & 12 & 0.57 & 0.12 \\
\hline 30598 & 18 & 0.82 & 0.08 & 0.54 & 0.01 & 303 & 1 & 0.63 & & 7 & 0.53 & 0.16 \\
\hline 30653 & 17 & 1.01 & 0.11 & 0.81 & 0.02 & 164 & 3 & 0.58 & 0.08 & 9 & 0.55 & 0.09 \\
\hline 30711 & & & & & & & 2 & 0.47 & 0.03 & 2 & 0.57 & 0.11 \\
\hline 30719 & & & & & & & 2 & 0.58 & 0.09 & 2 & 0.55 & 0.00 \\
\hline 30751 & & & & & & & 2 & 0.60 & 0.06 & 2 & 0.49 & 0.09 \\
\hline 30933 & & & & & & & 1 & 0.60 & & 2 & 0.60 & 0.05 \\
\hline 31015 & 17 & 0.83 & 0.09 & 0.41 & 0.01 & 244 & 1 & 0.52 & & 8 & 0.54 & 0.17 \\
\hline 31306 & 16 & 1.34 & 0.18 & 0.57 & 0.02 & 151 & 2 & 0.52 & 0.06 & 11 & 0.52 & 0.13 \\
\hline 31617 & 17 & 1.11 & 0.27 & 0.68 & 0.02 & 131 & 3 & 0.49 & 0.13 & 9 & 0.51 & 0.15 \\
\hline 31665 & & & & & & & 2 & 0.57 & 0.08 & 2 & 0.53 & 0.05 \\
\hline 31803 & 18 & 1.37 & 0.32 & 0.61 & 0.03 & 100 & 3 & 0.51 & 0.06 & 10 & 0.51 & 0.08 \\
\hline 31845 & 18 & 1.29 & 0.11 & 0.75 & 0.02 & 150 & 3 & 0.57 & 0.08 & 11 & 0.56 & 0.10 \\
\hline 32055 & 18 & 1.06 & 0.14 & 0.72 & 0.01 & 204 & 1 & 0.47 & & 7 & 0.54 & 0.09 \\
\hline 32121 & 17 & 1.17 & 0.13 & 0.76 & 0.01 & 216 & 1 & 0.47 & & 10 & 0.54 & 0.10 \\
\hline 32151 & & & & & & & 2 & 0.47 & 0.03 & 2 & 0.61 & 0.01 \\
\hline 32317 & & & & & & & 2 & 0.53 & 0.00 & 2 & 0.58 & 0.07 \\
\hline 32347 & 17 & 0.95 & 0.10 & 0.88 & 0.02 & 181 & 1 & 0.61 & & 8 & 0.57 & 0.16 \\
\hline 32555 & 18 & 1.07 & 0.10 & 0.81 & 0.02 & 133 & 3 & 0.55 & 0.03 & 10 & 0.54 & 0.08 \\
\hline 32700 & 18 & 0.87 & 0.10 & 0.84 & 0.01 & 238 & 1 & 0.52 & & 10 & 0.58 & 0.18 \\
\hline 32724 & & & & & & & 1 & 0.50 & & 2 & 0.50 & 0.00 \\
\hline 32835 & 14 & 0.77 & 0.07 & 0.56 & 0.01 & 215 & 1 & 0.60 & & 7 & 0.54 & 0.19 \\
\hline 32871 & 18 & 0.89 & 0.15 & 0.96 & 0.02 & 137 & 3 & 0.45 & 0.06 & 9 & 0.58 & 0.12 \\
\hline 32874 & & & & & & & 2 & 0.53 & 0.08 & 2 & 0.49 & 0.03 \\
\hline 32968 & & & & & & & 2 & 0.53 & 0.08 & 2 & 0.48 & 0.04 \\
\hline 32988 & 18 & 1.07 & 0.25 & 0.44 & 0.02 & 135 & 1 & 0.54 & & 5 & 0.57 & 0.09 \\
\hline 33069 & 16 & 1.20 & 0.08 & 0.77 & 0.02 & 174 & 3 & 0.56 & 0.13 & 9 & 0.52 & 0.16 \\
\hline
\end{tabular}

Notes. ${ }^{(a)}$ Number of $\mathrm{CN}$ features used to derive $[\mathrm{N} / \mathrm{Fe}] .{ }^{(b)}$ Errors in the abundance of Al due to errors in flux. ${ }^{(c)}$ Number of lines. 
E. Carretta et al.: Aluminium abundances in 47 Tuc and M 4

Table 3. continued.

\begin{tabular}{|c|c|c|c|c|c|c|c|c|c|c|c|c|}
\hline Star & $\mathrm{nr}^{a}$ & {$[\mathrm{~N} / \mathrm{Fe}]$} & $\mathrm{rms}$ & {$[\mathrm{Al} / \mathrm{Fe}]$} & $\mathrm{err}^{b}$ & $\mathrm{~S} / \mathrm{N}$ & $n^{c}$ & {$[\mathrm{Mg} / \mathrm{Fe}]$} & $\mathrm{rms}$ & $n^{c}$ & {$[\mathrm{Si} / \mathrm{Fe}]$} & $\mathrm{rms}$ \\
\hline 33195 & & & & & & & 1 & 0.62 & & 2 & 0.48 & 0.06 \\
\hline 33414 & 18 & 0.97 & 0.16 & 0.65 & 0.02 & 156 & 1 & 0.52 & & 10 & 0.53 & 0.21 \\
\hline 33617 & 18 & 1.03 & 0.13 & 0.87 & 0.01 & 264 & 3 & 0.43 & 0.01 & 11 & 0.51 & 0.09 \\
\hline 33629 & 17 & 1.30 & 0.16 & 0.69 & 0.03 & 98 & 2 & 0.48 & 0.05 & 12 & 0.52 & 0.09 \\
\hline 33683 & 18 & 0.90 & 0.17 & 0.60 & 0.01 & 230 & 1 & 0.56 & & 10 & 0.52 & 0.18 \\
\hline 33788 & & & & & & & 2 & 0.60 & 0.04 & 2 & 0.48 & 0.02 \\
\hline 33900 & 17 & 1.09 & 0.09 & 0.84 & 0.02 & 204 & 3 & 0.50 & 0.16 & 9 & 0.54 & 0.16 \\
\hline 33946 & & & & & & & 3 & 0.56 & 0.03 & 11 & 0.60 & 0.07 \\
\hline 34005 & 17 & 1.41 & 0.15 & 0.74 & 0.02 & 170 & 3 & 0.57 & 0.08 & 12 & 0.55 & 0.10 \\
\hline 34006 & 18 & 1.02 & 0.13 & 0.96 & 0.02 & 157 & 3 & 0.54 & 0.03 & 9 & 0.59 & 0.09 \\
\hline 34130 & 17 & 1.10 & 0.14 & 0.72 & 0.02 & 174 & 3 & 0.55 & 0.12 & 12 & 0.54 & 0.12 \\
\hline 34240 & 17 & 0.84 & 0.11 & 0.75 & 0.01 & 378 & 1 & 0.53 & & 9 & 0.55 & 0.10 \\
\hline 34502 & 18 & 1.06 & 0.20 & 0.81 & 0.02 & 158 & 3 & 0.59 & 0.29 & 11 & 0.55 & 0.21 \\
\hline 34726 & 18 & 1.17 & 0.13 & 0.76 & 0.01 & 227 & 3 & 0.60 & 0.01 & 10 & 0.51 & 0.15 \\
\hline 34907 & 17 & 1.34 & 0.28 & 0.75 & 0.02 & 142 & 3 & 0.56 & 0.09 & 10 & 0.53 & 0.09 \\
\hline 35022 & 17 & 1.23 & 0.12 & 0.68 & 0.02 & 178 & 3 & 0.56 & 0.06 & 11 & 0.52 & 0.11 \\
\hline 35061 & 18 & 0.96 & 0.21 & 0.66 & 0.04 & 81 & 3 & 0.58 & 0.09 & 12 & 0.60 & 0.10 \\
\hline 35455 & 18 & 0.90 & 0.11 & 0.76 & 0.02 & 195 & 3 & 0.58 & 0.12 & 6 & 0.58 & 0.14 \\
\hline 35508 & 17 & 1.27 & 0.15 & 0.72 & 0.03 & 94 & 3 & 0.55 & 0.01 & 11 & 0.55 & 0.13 \\
\hline 35571 & 17 & 0.86 & 0.22 & 0.50 & 0.02 & 150 & 3 & 0.59 & 0.05 & 12 & 0.53 & 0.11 \\
\hline 35627 & 17 & 0.89 & 0.25 & 0.77 & 0.02 & 137 & 1 & 0.51 & & 8 & 0.51 & 0.10 \\
\hline 35688 & 18 & 1.04 & 0.16 & 0.83 & 0.02 & 179 & 3 & 0.50 & 0.05 & 12 & 0.54 & 0.10 \\
\hline 35774 & 18 & 0.95 & 0.10 & 0.82 & 0.01 & 273 & 1 & 0.51 & & 5 & 0.56 & 0.06 \\
\hline 36356 & 18 & 1.23 & 0.16 & 0.83 & 0.02 & 162 & 1 & 0.60 & & 10 & 0.57 & 0.24 \\
\hline 36929 & & & & & & & 2 & 0.56 & 0.06 & 2 & 0.52 & 0.01 \\
\hline 36942 & 16 & 1.00 & 0.11 & 0.81 & 0.01 & 213 & 3 & 0.61 & 0.10 & 11 & 0.56 & 0.13 \\
\hline 37215 & 18 & 1.10 & 0.15 & 0.59 & 0.02 & 134 & 3 & 0.55 & 0.06 & 12 & 0.58 & 0.12 \\
\hline 38075 & 16 & 1.25 & 0.15 & 0.81 & 0.01 & 262 & 3 & 0.51 & 0.12 & 9 & 0.50 & 0.09 \\
\hline 38383 & 18 & 0.85 & 0.09 & 0.54 & 0.01 & 237 & 3 & 0.58 & 0.07 & 12 & 0.53 & 0.23 \\
\hline 38399 & 17 & 0.97 & 0.08 & 0.72 & 0.01 & 222 & 3 & 0.49 & 0.05 & 10 & 0.58 & 0.08 \\
\hline 38896 & 16 & 1.02 & 0.20 & 0.68 & 0.02 & 137 & 3 & 0.58 & 0.04 & 10 & 0.52 & 0.15 \\
\hline 40126 & 18 & 0.97 & 0.06 & 0.65 & 0.02 & 125 & 3 & 0.57 & 0.18 & 6 & 0.59 & 0.13 \\
\hline 42216 & 18 & 1.05 & 0.08 & 0.85 & 0.01 & 265 & 3 & 0.58 & 0.06 & 10 & 0.53 & 0.20 \\
\hline 42620 & 17 & 1.06 & 0.08 & 0.85 & 0.02 & 176 & 3 & 0.54 & 0.10 & 11 & 0.58 & 0.18 \\
\hline 42949 & 18 & 0.89 & 0.12 & 0.62 & 0.02 & 189 & 3 & 0.54 & 0.11 & 12 & 0.58 & 0.11 \\
\hline 43370 & 17 & 1.24 & 0.18 & 0.83 & 0.03 & 132 & 3 & 0.54 & 0.08 & 11 & 0.51 & 0.19 \\
\hline 44243 & 15 & 1.21 & 0.12 & 0.78 & 0.03 & 113 & 3 & 0.42 & 0.06 & 12 & 0.51 & 0.10 \\
\hline 44595 & 18 & 1.20 & 0.16 & 0.88 & 0.02 & 162 & 3 & 0.57 & 0.22 & 12 & 0.59 & 0.18 \\
\hline 44616 & & & & & & & 2 & 0.51 & 0.02 & 2 & 0.51 & 0.05 \\
\hline 45163 & 17 & 1.12 & 0.13 & 0.78 & 0.02 & 168 & 3 & 0.61 & 0.10 & 11 & 0.56 & 0.14 \\
\hline 45895 & 17 & 0.83 & 0.13 & 0.73 & 0.03 & 122 & 3 & 0.56 & 0.07 & 7 & 0.51 & 0.14 \\
\hline
\end{tabular}

